\title{
An in vivo approach to characterize novel variants associated with musculoskeletal disorders
}

Jennifer McAdow* ${ }^{* 1}$, Shuo Yang ${ }^{* 1}$, Tiffany Ou ${ }^{1}$, Gary Huang ${ }^{1,5}$, Matthew B. Dobbs ${ }^{6}$, Christina A. Gurnett $^{2,3,4}$, and Aaron N. Johnson ${ }^{1,7}$

*these authors contributed equally to this work

${ }^{1}$ Department of Developmental Biology,

${ }^{2}$ Department of Neurology,

${ }^{3}$ Department of Orthopedic Surgery,

${ }^{4}$ Department of Pediatrics,

Washington University in St. Louis, St. Louis, MO, USA.

${ }^{5}$ Current address: Graduate Program in Genetics and Genomics, Baylor College of Medicine

${ }^{6}$ Paley Orthopedic and Spine Institute, West Palm Beach, FL, USA

${ }^{7}$ Author for correspondence: anjohnson@wustl.edu

Running Title: TPM2 variants disrupt myogenesis 


\section{Summary}

Nemaline Myopathy (NM) is the most common congenital myopathy, characterized by

3 extreme weakness of the respiratory, limb, and facial muscles. Pathogenic variants in

4 Tropomyosin 2 (TPM2), which encodes a skeletal muscle specific actin binding protein essential

5 for sarcomere function, cause a spectrum of musculoskeletal disorders that include NM as well

6 as Cap Myopathy, congenital fiber type disproportion, and distal arthrogrypsosis (DA). TPM2-

7 related disorders have not been modeled in vivo, so we expressed a series of dominant,

8 pathogenic TPM2 variants in Drosophila embryos and found two variants, K49Del and E122K,

9 significantly affected muscle morphogenesis and muscle function, in part by disrupting myotube

10 guidance. Transient overexpression of K49Del and E122K also disrupted the morphogenesis of

11 immortalized mouse myoblasts in vitro, and negatively affected zebrafish muscle development

12 and function in vivo. We used our transient overexpression assays in zebrafish to characterize

13 two novel TPM2 variants that we identified in DA patients (V129A and E139K), and found these

14 variants caused musculoskeletal defects similar to those of the known pathogenic variants. In

15 addition, the severity of musculoskeletal phenotypes in zebrafish expressing TPM2 variants

16 correlated with the severity of clinical phenotypes observed in DA patients. Our study

17 establishes transient overexpression in zebrafish as an efficient platform to characterize variants

18 of uncertain significance in TPM2 in vivo, and suggests that this method can be used to predict

19 the clinical severity of variants associated with DA and congenital myopathies. 
Introduction

Tropomyosins are obligate actin binding proteins that form hetero- and homodimers (Hardeman et al., 2020). Head-to-tail tropomyosin polymers assemble along the length of actin filaments, and in the sarcomere tropomyosin regulates contractility by controlling the ability of thick filament myosin to access actin thin filaments (Squire et al., 2017). To initiate muscle contraction, $\mathrm{Ca}^{2+}$ released from the sarcoplasmic reticulum binds to sarcomeric troponin, which alters thin filament confirmation. The intermediate thin filament confirmation allows myosin to contact actin and further displace tropomyosin to drive maximal thin filament sliding and complete contraction (Squire et al., 2017).

Tropomyosin is encoded by four loci in humans (TPM1, TPM2, TPM3, and TPM4), with TPM2 and TPM3 being the predominant skeletal muscle isoforms (Hardeman et al., 2020). Pathogenic TPM2 variants are causative of congenital skeletal muscles diseases, and much attention has been given toward understanding how TPM2 variants disrupt sarcomere function. However, tropomyosin also functions outside of the sarcomere to regulate cytoskeletal changes that drive cell migration and cellular metastasis (Bugyi et al., 2010; Lees et al., 2013; Shin et al., 2017). Since skeletal muscle development depends on cytoskeletal dynamics to direct muscle precursor migration (Wang et al., 2018) and myofiber morphogenesis (Williams et al., 2015; Yang et al., 2020), it is distinctly possible that TPM2 variants adversely affect cytoskeletal dynamics prior to sarcomere assembly which could disrupt overall muscle myogenesis.

Congenital diseases associated with TPM2 include Nemaline Myopathy (NM) and Cap Myopathy (CM), which are both associated with extreme muscle weakness (hypotonia)(Clarke et al., 2009; Davidson et al., 2013; Mokbel et al., 2013; Ohlsson et al., 2008; Tajsharghi et al., 2007b). The diagnostic features for NM and CM are the presence of nemaline bodies and caplike structures on muscle biopsy. Pathogenic TPM2 variants are also causative of congenital fiber type disproportion (CFTD), in which highly oxidative type 1 myofibers are predominant and heterogeneity of DA clinical phenotypes has necessitated subtype classifications with hierarchical criteria (Bamshad et al., 2009). TPM2 variants are causative of DA type 1 (DA1)(Sung et al., 2003), which is characterized by contractures of the hands and feet including permanently bent fingers (camptodactyly) and clubfoot (talipes equinovarus) (Bamshad et al.,

51 2009). TPM2 variants are also associated with DA type 2B (DA2B) (Li et al., 2018; Tajsharghi et

52 al., 2007a), which is characterized by facial abnormalities in addition to contractures of the extremities (Bamshad et al., 2009). DA patients often show hypotonia (Marttila et al., 2014; 
54 Mroczek et al., 2017), arguing skeletal muscle dysfunction contributes to the overall disease 55 mechanism.

TPM2 variants are also causative of Escobar variant of multiple pterygium syndrome (EVMPS)(Marttila et al., 2014; Tajsharghi et al., 2012; Vogt et al., 2020). EVMPS patients show joint contractures similar to those reported for DA patients, but EVMPS is distinguished from DA by the presence of webbing (pterygia) at the neck, elbows, or knees (Morgan et al., 2006). It is important to note that hypotonia often extends to the diaphragm in patients carrying TPM2 variants, which may require lifelong respiratory intervention (Clarke and North, 2003; Marttila et al., 2014; Ohlsson et al., 2008). The broad spectrum of clinical phenotypes associated with TPM2 mutations has obscured a clear understanding as to how pathogenic TPM2 variants disrupt skeletal muscle form and function.

While the in vivo disease mechanisms that underlie TPM2 associated disorders are incompletely understood, the inheritance of TPM2 congenital diseases follows an autosomal dominant pattern (Bamshad et al., 1994; Mokbel et al., 2013). One notable exception would be the pathogenic variant Q210*, which was shown to be autosomal recessive in a consanguineous family with EVMPS (Monnier et al., 2009; Tajsharghi et al., 2012). Over 30 pathogenic TPM2 variants have been reported, and the variants themselves show a fairly even distribution along the protein (Fig. 1A)(Marttila et al., 2014). TPM2 is comprised of 7 quasi repeats, each divided into one $\alpha$-sheet and one $\beta$-sheet, with one residue per quasi repeat binding actin (Marston et al., 2013). In addition to the quasi repeats, TPM2 forms a coiled-coil that follows the typical heptad repeat of seven residues, labeled $a-g$, where $b$ and $f$ residues interact with actin and $g$ residues are charged. Surprisingly, only one pathogenic variant changes an actin-binding residue (K128E)(Marttila et al., 2014), while 7 variants cluster to charged $g$ positions (Fig. 1B). The molecular genetics of TPM2-related disorders argues that pathogenic TPM2 variants are dominant, gain-of-function mutations that indirectly disrupt tropomyosin-actin interactions.

Extensive biochemical studies have been used to understand the gain-of-function phenotypes induced by TPM2 variants. Thin filaments, or even entire muscle fibers, can be reconstituted in vitro to assay myosin driven actin motility (Avrova et al., 2018; Borovikov et al., 2020; Borovikov et al., 2017a; Borovikov et al., 2017b; Karpicheva et al., 2020; Marston et al., 2013; Ochala et al., 2010). Reconstituted thin filaments contain actin, tropomyosin, and troponin, such that actin motility can be measured in response to a $\mathrm{Ca}^{2+}$ gradient. Actin motility assays have shown that some TPM2 variants increase $\mathrm{Ca}^{2+}$ sensitivity, causing maximum actin 
$88 \mathrm{Ca}^{2+}$ sensitivity (Table 1)(Borovikov et al., 2015; Marston et al., 2013; Marttila et al., 2012; 89 Ochala et al., 2010). The addition of fluorescent probes and proteins to actin motility assays 90 revealed that the $\mathrm{Ca}^{2+}$ sensitivity of TPM2 variants correlates with the ability of troponin and 91 myosin to shift tropomyosin away from actin, and that pathogenic substitutions alter tropomyosin 92 flexibility (Avrova et al., 2018; Borovikov et al., 2020; Borovikov et al., 2017a; Borovikov et al., 93 2017b; Karpicheva et al., 2020). Since tropomyosin often exists as a heterodimer, TPM2 94 variants likely act as gain-of-function mutations by altering $\mathrm{Ca}^{2+}$ sensitivity when dimerized with 95 wild-type isoforms (Avrova et al., 2018; Borovikov et al., 2020; Matyushenko et al., 2019). 96 Despite these extensive studies into the biochemical properties of TPM2 mutations, pathogenic TPM2 variants have yet to be characterized in vivo.

We set out to model TPM2 congenital disorders in vivo, with the prediction that TPM2 mutations would adversely affect muscle development and function. TPM2 has been deleted in mice, and heterozygotes showed compromised lens regeneration (Shibata et al., 2018). However, genome-edited TPM2 variants have not been reported in any organism. 28 pathogenic TPM2 variants affecting the coding region have previously been identified, and we used transgenic overexpression in Drosophila and zebrafish embryos to study the effects of known variants on embryonic and larval muscle phenotypes. These studies showed that transient overexpression could be useful to model the pathogenic effects of TPM2 variants, so we expressed three variants that we identified in patients with musculoskeletal disorders in zebrafish. The variants V129A, E139K, and A155T caused phenotypes similar to those we identified for known pathogenic variants, which provided additional evidence that the variants are in fact pathogenic. In addition, the strength of phenotypes from our transient overexpression assays in fish correlated with the severity of patient phenotypes, suggesting our disease model has the power to predict the clinical severity of newly identified variants. 


\section{Results}

We set out to model TPM2 related diseases in vivo by characterizing a panel of variants that represent the key characteristics of the spectrum of TPM2 pathogenic variants. A set of 8 variants in highly conserved residues are causative of the 5 TPM2 associated disorders, including NM (K7Del, E41K, R133W), CM (K49Del, N202K), CFTD (E122K, R133P), DA (K7Del, E41K, R91G, R133W), and EVMPS (R133W; Table 1). The representative variants are also equally distributed between $\alpha$-sheets (K7Del, K49Del, R91G, R133P/W) and $\beta$-sheets variants generally cluster to $b, f$, and $g$ residues (Fig. 1B). The 8 representative variants clustered to the $f(E 41 K, N 202 K)$ and $g$ (K7Del, K49Del, R91G, E122K, R133P) positions, which is consistent with the overall distribution of pathogenic variants along the coiled-coil heptad (Fig. 1B). The variants K7Del, E41K, K49Del, R91G, E122K, R133P, R133W, and N202K are thus a representative collection of TPM2 mutations causative of congenital disease.

TPM2 variants disrupt myogenesis

Tropomyosin 2 (Tm2) is the Drosophila orthologue of human TPM2, and the two proteins show a high degree of sequence conservation (Fig. 1A). Overexpression studies in Drosophila have successfully modeled pathogenic variants in MYH3 associated with DA (Guo et al., 2020), so we used the binary UAS-GAL4 system to express Drosophila Tm2, wild-type human TPM2, and the set of 8 human TPM2 variants in Drosophila embryonic muscle precursors (Fig. 1C). For these experiments, UAS constructs were targeted to a common genomic landing site to minimize mRNA expression differences among the variants. The GAL4 lines slou.Gal4 and nau.Gal4 direct UAS transgene expression in a subset of muscle precursors (Yang et al., 2020), which allowed us to quantify muscle morphology at single cell resolution (Fig. 1C). Embryonic muscles are named by their position and orientation in the segment, and the Longitudinal Oblique 1 (LO1) muscle shows a striking oblique morphology (Fig. 2A). LO1 muscles that expressed GFP-tagged TPM2 variants under the control of slou.Gal4 showed several abnormalities including rounded and generally misshapen morphologies, and attachments to the wrong tendon (Fig. 2A,B). Variant expressing LO1 muscles also failed to develop in the correct position, and were sometimes missing by the end of myogenesis (Fig. 2A,B). The frequency of LO1 muscle phenotypes was higher in muscles that expressed TPM2 variants than in muscles that expressed wild-type TPM2 or Drosophila Tm2, and LO1 muscles that expressed K49Del

144 showed the highest frequency of muscle defects within the set of 8 pathogenic variants (Fig. 
the control of nau.Gal4 were significantly shorter than VO5 muscles that expressed wild-type TPM2 or Tm2 (Fig. 2C,D). Among the 8 variants tested, VO5 muscles that expressed E122K showed the strongest phenotype (Fig. 2C,D).

Our transgenic overexpression studies argue K49Del and E122K act as strong gain-offunction alleles in vivo. K49Del deletes a single residue in an $\alpha$-zone and is associated with CM, while E122K is a substitution in a $\beta$-zone and is associated with CFTD (Fig. 1A, Table 1). Surprisingly, neither the position of the variant along the TPM2 protein nor the clinical diagnosis associated with the mutation correlated with the strength of the myogenic phenotype. In addition, the phenotypes we identified in TPM2 expressing muscles occurred prior to sarcomere assembly, suggesting pathogenic TPM2 variants affect muscle development and function through sarcomere independent pathways.

\section{K49Del disrupts myotube guidance}

Cellular guidance is a morphogenetic process in which a cell remains spatially fixed, and extends long processes to interact with other cells. During myotube guidance in Drosophila, a nascent myotube extends bilateral projections toward tendon cells at the segment border to establish overall muscle morphology. The phenotypes we observed in K49Del expressing LO1 muscles, including short rounded muscles and muscles attached to the wrong tendon, are consistent with defects in myotube guidance (Fig. 2A)(Yang et al., 2020). We have correlated actin dynamics with proper myotube guidance (Williams et al., 2015; Yang et al., 2020), and we suspected that K49Del muscle phenotypes are due to myotube guidance defects.

Live imaging of nascent LO1 myotubes revealed the primary LO1 leading edge initially elongates dorsally, nearly parallel to the dorsal/ventral axis, until it reaches the medial-posterior of the hemisegment (Movie 1). The leading edge then makes a dramatic turn toward the anterior and elongates parallel to the anterior/posterior axis until it reaches a tendon cell at the medial-anterior edge of the hemisegment. The secondary LO1 myotube leading edge elongates a short distance toward ventral-posterior side of the hemisegment where it attaches to a second tendon cell, ultimately giving the LO1 muscle its characteristic oblique morphology.

LO1 myotubes that expressed K49Del showed several unusual behaviors. Some K49Del expressing myotubes would initiate elongation, but both leading edges would retract and show a rounded muscle phenotype at the end of myogenesis (Movie 1). In other examples, the primary and secondary leading edges in K49Del expressing myotubes would elongate appropriately, but the lateral membrane would form a third leading edge and elongate to a third tendon cell (Movie 2). Muscles attached to 3 tendons would presumably be unable to move the exoskeleton 
correctly. The K49Del variant therefore disrupts myotube guidance, which adversely affects muscle development prior to sarcomere assembly.

\section{K49Del and E122K inhibit muscle function in vivo}

After embryogenesis, Drosophila larva develop through three distinct stages (L1-L3), which are characterized by massive muscle hypertrophy (Demontis and Perrimon, 2009). We used Mef2.Gal4 to express K49Del and E122K in all embryonic and larval muscles, and assayed larval muscle morphology and function. Variant expressing muscles were significantly longer in live L3 larva, which could reflect a reduced contractile state (Fig. 3A,B). Standardized L3 larva locomotion assays have been developed to assess muscle function in insects (Brooks et al., 2016), and we found that K49Del and E122K expressing L3 larva had reduced locomotor activity compared to TPM2 expressing controls (Fig. 3C).

We generated UAS constructs encoding untagged TPM2 proteins that were targeted to a common genomic landing site for rescue experiments with a null allele of Drosophila Tm2, $T m 2^{D 8-261}$ (Williams et al., 2015). Tm2 $2^{D 8-261}$ homozygous mutants survive to the end of embryogenesis, but $42.1 \%$ failed to hatch presumably due to compromised muscle function (Fig. 3D). We used embryo hatching frequency to assay tropomyosin function, and found wildtype TPM2 improved Tm2 $2^{D 8-261}$ embryo hatching (Fig. 3D). However, the hatching frequency was not significantly different between TPM2 and K49Del rescued embryos (Fig. 3D). We repeated the rescue experiments with Drosophila Tm2, and found similar results (Fig. 3D), except $T m 2^{D 8-261}$ larva that expressed Tm2.K49Del often lacked muscles, and the remaining Tm2.K49Del expressing muscles appeared longer than controls (Fig. 3E). Tm2 $2^{D 8-261}$ homozygous animals that hatched showed complete lethality during the L1 stage, but neither wild-type TPM2 nor K49Del significantly rescued $T m 2^{D 8-261} \mathrm{~L} 1$ lethality. Overall our larval studies confirmed that pathogenic TPM2 variants inhibit muscle function in vivo, but the lack of a complete $T m 2^{D 8-261}$ rescue prompted us to model TPM2 related diseases in complementary systems.

\section{TPM2 variants disrupt myotube morphogenesis in vitro}

To determine if pathogenic TPM2 variants also disrupt muscle morphology in vertebrates, we expressed K49Del and E122K in C2C12 cells, which are immortalized mouse myoblasts capable of differentiating into multinucleate myotubes. Under differentiation conditions, $\mathrm{C} 2 \mathrm{C} 12$ myoblasts fuse and form nascent myotubes that extensively elongate (Blau et al., 1983). K49Del and E122K proteins mislocalize in C2C12 cells (Abdul-Hussein et al., 
214 2013), but the phenotypes of variant expressing myotubes were not previously characterized.

215 C2C12 cells that expressed untagged, wild-type TPM2 were morphologically similar to control

216 treated cells after 7 days of differentiation, but cells that expressed K49Del and E122K showed

217 significantly reduced myoblast fusion and myotube elongation (Fig. 4A-D). The K49Del and

218 E122K variants therefore disrupt muscle morphogenesis in mammalian cells. Since C2C12 cells

219 develop independent of other musculoskeletal tissues, our studies argue that TPM2 related disease mechanisms act cell autonomously on developing myofibers.

\section{A transient overexpression assay of variant pathogenicity in zebrafish}

Our studies in mammalian cells supported the hypothesis that pathogenic TPM2 variants disrupt muscle morphogenesis in vertebrates. The K49 and E122 residues are conserved in zebrafish TPM2 (Fig. 5A), and we recently modeled DA2A in zebrafish (Whittle et al., 2020). Genome edited fish heterozygous for the pathogenic variant $\mathrm{R} 672 \mathrm{H}$ in $M Y H 3$ showed musculoskeletal abnormalities consistent with joint contractures (Whittle et al., 2020). While the efficiency of genome editing technologies in zebrafish is continuing to improve, the injection of variant-encoding capped mRNAs into fertilized embryos is a well-established tool for rapidly evaluating variant pathogenicity in developing embryos and larva (Jing and Zon, 2011). Though this technique is difficult to use for large transcripts, we took advantage of the comparatively small TPM2 coding sequence to generate and inject a gradient of mRNA concentrations into one-cell stage embryos and assess muscle morphology at $26 \mathrm{hr}$ post fertilization (hpf; Fig. 5BD).

To validate our transient overexpression assay, we injected embryos with a standardized dose of mRNA (600pg), and quantified muscle and tendon phenotypes in larva that expressed pathogenic or benign variants (Fig. 6A,B). The injection of wild type TPM2 mRNA or E273K mRNA (rs3180843, LOVD variant 0000446934), which encodes a benign variant identified in a patient with normal muscle function, had negligible effects on myofiber and somite length in the trunk. However, injection of K49Del and E122K encoding mRNAs induced dose dependent defects on musculoskeletal morphology (Fig. 5D, 6A). Larva that expressed K49Del and E122K had significantly shorter slow-twitch myofibers than E273K expressing larva (Fig. 6C). In addition, the slow-twitch myofibers were disorganized, with the myofiber ends often clustered at the somite boundary or even in the center of the somite (Fig. 6A). K49Del expressing larva had

246 larva had significantly shorter somite lengths (Fig. 6C). K49Del and E122K did not appear to 247 affect fast-twitch myofiber morphology. 
Myofibers in the trunk attach to tendons in the myosepta, which are located along the somite boundaries and strongly express the tendon structural protein thrombospondin (THBS4) (Fig. 6B). Larva that expressed K49Del and E122K showed multiple tendon phenotypes that included tendons incorrectly positioned in the center of the somite, bifurcated myosepta, and myosepta with broken or incomplete expression of thrombospondin (Fig. 6B). While both

253 K49Del and E122K caused tendon defects, only E122K larva had a statistically significantly 254 higher frequency of tendon defects than E273K larva (Fig. 6E). Taken together, our studies 255 show that the musculoskeletal phenotypes produced by the transient overexpression of TPM2 alleles can be used to statistically distinguish pathogenic from benign variants. In addition, our overexpression assays show that K49Del and E122K disrupt musculoskeletal system morphogenesis in zebrafish similar to what we observed in Drosophila. These studies provide further evidence that pathogenic TPM2 variants directly affect myogenesis.

\section{Novel and recurring TPM2 variants identified in DA patients}

Having established a functional assay in zebrafish that can quantitatively identify pathogenic variants in TPM2, we decided to evaluate variants of uncertain significance identified in patients with pediatric musculoskeletal disorders treated at Washington University. Patient I, with isolated bilateral clubfoot but no hand contractures, has a heterozygous TPM2 V129A variant. Patient II and III are unrelated DA1 patients with single heterozygous TPM2 variants (E139A and A155T; Fig. 7B,C). Patient III also had mild distal lower extremity weakness and fatigue upon running. There was no family history of arthrogryposis or clubfoot, however parents were unavailable for genotyping. Two of the TPM2 variants, V129A and E139K, had not previously been identified in patients with myopathies or arthrogryposis. The third variant, A155T, was previously identified in a Chinese family with DA1 (Jin et al., 2017). The residues affected by V129A, E139K, and A155T are conserved to zebrafish (Fig. 5A), so we used our transient overexpression assay to provide additional evidence for pathogenicity.

Novel TPM2 variants disrupt muscle development and muscle function in zebrafish.

Larva that expressed V129A, E139K, and A155T showed defects in musculoskeletal

277 morphogenesis similar to those that expressed K49Del and E122K (Fig. 8A,B). Slow-twitch

278 fibers from A155T expressing larva were significantly shorter and significantly fewer than E273K 279 controls, and A155T somites were significantly smaller (Fig. 8C,D). Larva that expressed V129A 280 and E139K had disorganized myofibers, and V129A myofibers were significantly shorter than controls (Fig. 8A,C). Larva that expressed E139K also showed a significant decrease in somite 
282 size (Fig. 8C). In addition, larva that expressed V129A, E139K, and A155T showed a significant 283 increase in the frequency of tendon phenotypes compared to E273K controls (Fig. 8E).

284 The startle response in zebrafish larva is a well-characterized reflex used to assay motor 285 function, and we previously showed muscle function is compromised in a MYH3 model of DA 286 using the larval startle response (Whittle et al., 2020). To understand if TPM2 variants affect 287 muscle function in zebrafish, we injected mRNAs into one-cell stage embryos, and ran 288 automated tracking assays in larva 6 days post-fertilization (dpf) (Fig. 5B, 9A). After a stimulus, 289 the startle response induces a reflexive swim behavior that is quantified by distance swam and 290 the escape velocity. K49Del and E122K expressing larva showed an altered startle response, 291 but surprisingly the swim distance and escape velocity of K49Del and E122K larva were not 292 significantly different than E273K expressing larva (Fig. 9B). However, A155T expressing larva 293 showed significantly reduced swim distance and escape velocity compared to E273K 294 expressing larva, and V129A larva showed significantly reduced escape velocity (Fig. 9B). 295 E139K expressing larva showed an affected startle response, but the differences were not 296 significantly different than E273K expressing larva (Fig. 9B). Nevertheless, the TPM2 variants 297 we identified in DA patients caused defects in muscle morphogenesis and muscle function, with 298 A155T causing the most severe phenotypes. Taken together, our transient overexpression 299 assays provide additional evidence that the novel TPM2 variants V129A and E139K are 300 pathogenic, while further confirming A155T is causative of DA. 


\section{Discussion}

To date, over 30 TPM2 variants have been identified in patients with myopathies and arthrogryposis, but the biochemical properties of only a few variants have been tested.

304 Furthermore, in vivo studies investigating the physiological consequences of pathogenic TPM2 305 variants were completely lacking. We expressed TPM2 variants in multiple model systems, and 306 found pathogenic variants disrupt muscle development and muscle function. By focusing on two pathogenic variants, we developed a transient overexpression assay in zebrafish that benchmarked histological phenotypes and functional phenotypes of pathogenic variants against a known benign variant. Clinical sequencing of DA patients identified two novel TPM2 variants, $\mathrm{V} 129 \mathrm{~A}$ and $\mathrm{E} 139 \mathrm{~K}$, and a recurring variant, $\mathrm{A} 155 \mathrm{~T}$, which we tested in our transient overexpression assay. V129A, E139K, and A155T caused musculoskeletal defects similar to those of the known pathogenic variants, and our analyses provide support for pathogenicity of all three variants. Strikingly, A155T was the only variant to cause statistically significant phenotypes in every assay we developed, and the clinical symptoms in the patient with the A155T variant were the most severe among the patients in our study. These results argue that our transient overexpression assay in zebrafish can efficiently characterize variants of uncertain significance identified in patients with musculoskeletal disorders.

Investigations of TPM2 related disease mechanisms have largely been focused on understanding the role of TPM2 in the sarcomere. Thin filament motility assays uncovered the biochemical properties of TPM2 variants in response to $\mathrm{Ca}^{2+}$, and the pathogenic variants tested so far have shown increased as well as reduced $\mathrm{Ca}^{2+}$ sensitivity (Table 1 ). The basis for $\mathrm{Ca}^{2+}$ sensitivity is thought to reside in the flexibility or rigidity of the TPM2 dimer, which correlates with the ability of troponin and myosin to shift tropomyosin away from actin (Avrova et al., 2018; Borovikov et al., 2020; Borovikov et al., 2017a; Borovikov et al., 2017b; Karpicheva et al., 2020). Here, we found that TPM2 variants disrupted muscle morphogenesis prior to sarcomere assembly in vivo. Our live imaging in Drosophila embryos showed K49Del expressing myotubes had elongation defects and used inappropriate muscle attachment sites to adhere to the exoskeleton (Movies 1,2). We observed similar myotube elongation defects in $\mathrm{C} 2 \mathrm{C} 12$ cells that expressed pathogenic TPM2 variants. Since C2C12 cells develop in the absence of positional cues from other tissues, our studies argue TPM2 disease mechanisms act cell autonomously to disrupt myofiber morphogenesis prior to sarcomere assembly.

Tropomyosin has well-documented roles outside of the sarcomere in non-muscle cells, most notably during cell migration. Dynamic changes to the cytoskeleton, coupled with changes in the expression of cell adhesion proteins, drive cell migration. Tropomyosins regulate the rate 
335 of actin polymerization and depolymerization (Bugyi et al., 2010; Janco et al., 2016; 336 Robaszkiewicz et al., 2016), so it is not surprising that TPM2 and TPM3 are required for single 337 cell as well collective cell migration (Lees et al., 2013; Shin et al., 2017). In vertebrates, 338 myoblasts specified in somites migrate to sites of muscle morphogenesis where they fuse to 339 form myotubes, which in turn elongate and attach to tenocytes (Dennis et al., 1981; Kardon, 340 1998). During zebrafish myogenesis, myoblasts that give rise to slow- and fast-twitch myofibers 341 are developmentally distinct. Slow myoblasts known as adaxial cells are specified medially, 342 nearest the notochord, and migrate radially to form elongated myotubes on the superficial, 343 outermost region of the somite (Keenan and Currie, 2019). Adaxial cell migration is dependent on cadherin-mediated adhesion, and the slow-twitch region of the myotome in larva that expressed K49Del, E122K, V129A, and A155T (Figs. 6B, 8B) bore a striking resemblance to the slow-twitch myotome of larva that expressed reduced levels of $n$-cadherin (Cortés et al., 2003). It is possible that pathogenic TPM2 variants disrupt adaxial cell migration in zebrafish, suggesting myoblast migration may be affected in patients with TPM2 related disorders. site of myogenesis. However, similar to vertebrates, Drosophila myoblasts will fuse to form myotubes that elongate and identify muscle attachment sites (Yang et al., 2020). Myotube elongation and attachment site selection are collectively known as myotube guidance, which is similar to axon guidance in many respects. Cellular guidance is distinct from cell migration because during both myotube guidance and axon guidance, the cell remains spatially fixed but generates long projections to interact with other cells. Myotube guidance depends on regulated cytoskeletal dynamics, particularly of the actin cytoskeleton (Williams et al., 2015; Yang et al., 2020). The TPM2 muscle morphogenesis defects we have observed in Drosophila, zebrafish, and cultured cells are likely the result of improperly regulated actin dynamics in migrating myoblasts or in elongating myotubes that expressed pathogenic variants. DA and amyoplasia (absence of muscle) were often thought to be distinct clinical diagnoses, but amyoplasia was recently reported in a case of congenital DA (Chong et al., 2020). Our studies provide additional support for a model in which DA variants disrupt muscle development. Zebrafish that expressed 363 the DA associated variants $\mathrm{E} 139 \mathrm{~K}$ and $\mathrm{A} 155 \mathrm{~T}$ had highly disorganized myofibers that could 364 result in less skeletal muscle mass (Fig. 8A,B). It will be important to develop stable knock in 365 models to characterize the effects of TPM2 pathogenic variants on myoblast migration, myotube 366 guidance, and long-term muscle homeostasis.

At present, 14 TPM2 variants have been identified in patients with myopathies and arthrogrypsosis in which the significance of the variant has not been definitively defined (Table 
2). The number of TPM2 variants with uncertain significance is likely to increase because these variants are being identified in patients with isolated clubfoot, which is much more common condition than myopathies or arthrogryposis. The incidence of clubfoot in the United States is

372 1:1000 live births, but the underlying causes are often unknown. One approach toward 373 understanding isolated clubfoot is to expand clinical sequencing, which will likely uncover novel 374 TPM2 variants. Phenotypic variability among patients with TPM2 variants can make genotype375 phenotype correlations difficult (Marttila et al., 2014), but the stringency of our benchmarked 376 transient overexpression assay unambiguously distinguishes pathogenic from benign variants. 377 In addition, the assay could be expanded to include other loci associated with myopathies and arthrogrypsosis, which may also uncover new variants that contribute to isolated clubfoot pathogenesis.

One unexpected validation of our transient overexpression assay was that A155T caused the most severe phenotype of any of the variants tested, which also correlated with the clinical severity. The correlation between our laboratory and clinical observations likely reflects the unbiased design of our overexpression assay. Histological phenotypes were quantified by physically measuring individual myofibers and somites in large numbers of larva, and the locomotor phenotypes were quantified automatically by imaging software. We also controlled for day-to-day variation in our assays by normalizing our results to wild-type injected larva each time we injected variants, which allowed us to compare the relative effect of each variant on phenotype severity. One exciting possibility is that our benchmarked transient overexpression assay will have the power to predict the clinical severity of TPM2 variants, although more variants need to be evaluated to determine the accuracy of our models.

\section{Acknowledgements}

393 We thank Sharon Amacher for sending $\operatorname{Tg}($ acta1:GFP) fish and the Washington University 394 School of Medicine Zebrafish Consortium for providing an outstanding environment to conduct 395 fish work. ANJ was supported by NIH R01AR070299 (NIAMS), and ANJ and CAG were 396 supported by NIH R03HD104065 (NICHD). Research reported in this publication was also 397 funded by the Eunice Kennedy Shriver National Institute of Child Health and Human 398 Development of the National Institutes of Health under Award number 3P50HD103525-01S1 to 399 the Intellectual and Developmental Disabilities Research Center at Washington University and 400 the Washington University Institute of Clinical and Translational Sciences Award number 401 UL1TR002345 from the National Center for Advancing Translational Sciences (NCATS), and R01AR067715-06 (CAG and MBD). 


\section{Author Contributions}

405 Conceptualization: A.N.J., C.A.G, J.M., S.Y.; Methodology: A.N.J., C.A.G, S.Y.; Formal analysis:

406 S.Y., J.M., G.H., C.A.G, A.N.J.; Investigation: S.Y., J.M., G.H., P.H., A.N.J.; Resources: C.A.G.,

407 A.N.J.; Data curation: S.Y., J.M., A.N.J.; Writing original draft: A.N.J., C.A.G. ; Visualization:

408 S.Y., A.N.J.; Supervision: S.Y., J.M., A.N.J.; Project administration: A.N.J.; Funding acquisition:

409 C.A.G., A.N.J. 


\section{Materials and Methods}

\section{Drosophila genetics}

The TPM2 and Tm2 transgenic variants were constructed by PCR cloning the TPM2

413 ORF (clone HsCD00368588, PlasmID) and the Tm2 ORF (RE15528, Berkeley Drosophila

414 Genome Project) into pEntr (Life Technologies), followed by recombination into destination 415 vector TWG (Drosophila Genome Resource Center) to add a C-terminus GFP tag. TWG clones 416 served as a template to generate variants by site directed mutagenesis as described (Johnson 417 et al., 2013). Tagged and untagged ORFs were PCR subcloned into pUASt.attB using $418 \mathrm{EcoRI} / \mathrm{Xbal}$. All constructs were targeted to the same attP site on chromosome 3L (65B2) using ФC31 integrase and standard injection methods (Rainbow Transgenic Flies, Inc.). pUASt.attB constructs were fully sequenced prior to injection.

Additional stocks used in this study were $P\{$ GMR40D04-GAL4attP2 (slou.Gal4), P\{GMR57C12-GAL4\}attP2 (nau.Gal4), Tm2 ${ }^{\text {D8-261 (Williams et al., 2015), P(lacZ-kirre }}{ }^{\text {rP298}) ~(N o s e ~}$ et al., 1998), and P(PTT-GC)Tm2 ZCL2456 (Buszczak et al., 2007). Cyo, P(wg.lacZ), Cyo, $P$ (twi.Gal4), P(UAS.GFP), TM3, P(ftz.lacZ), and TM3, P(twi.Gal4), P(UAS.GFP) balancers were used to identify homozygous embryos. Fly stocks were obtained from the Bloomington Stock Center unless otherwise referenced.

\section{Cell culture}

TPM2 mammalian expression constructs were generated by PCR subcloning variants from pUASt.attB into pCMV-IRES-eGFP (Addgene 78264) using Xbal/EcoR1. C2C12 cells were seeded in 6-well-plate format, grown in standard conditions to $60 \%$ confluency in growth medium (10\% FBS in DMEM), and transfected with $1 \mu \mathrm{g}$ of DNA per manufacturer's specifications (Lipofectamine 3000, L3000015, Thermofisher). Empty pCMV-IRES-eGFP was used as a control. Growth media was changed to differentiation media ( $2 \%$ horse serum in DMEM) 24hrs after transfection; cells were differentiated for 7 days and prior to fixation.

\section{Fish genetics and injections}

Danio rerio were maintained in accordance with approved institutional protocols under the supervision of the Institutional Animal Care and Use Committee (IACUC) of Washington University, which is fully accredited by the AAALAC. Wild-type zebrafish were from line AB. The $\operatorname{Tg}\left(\right.$ acta1:GFP) ${ }^{z f 13}$ line has been previously described (Higashijima et al., 1997). TPM2 variants were PCR subcloned from pUASt.attB into pCR2.1 (K202040, Thermofisher), capped RNAs were transcribed with a T7 mMessage mMachine kit (AM1344, Thermofisher), and embryos 
444 at $6 \mathrm{dpf}$ for functional assays (feeding protocols began at $4 \mathrm{dpf}$ ). Control injected larva were

445 collected to normalize each cohort.

446 Immunohistochemistry, imaging, and image quantification

447 Drosophila. Dechorionated embryos were fixed in $4 \%$ formaldehyde, devitellinated with 448 heptane/methanol, and antibody stained as described (Johnson et al., 2013). Antibodies used 449 were $\alpha$-Mef2 (1:1000, gift from R. Cripps), $\alpha$-Myosin Heavy Chain (1:600, Abcam, MAC147), $\alpha-$ 450 GFP (1:600, Torrey Pines Biolabs, TP-401), and $\alpha$ - $\beta$ gal (1:100, Promega, Z3781). HRP451 conjugated secondary antibodies in conjunction with the TSA system (Molecular Probes) were 452 used to detect primary antibodies.

453 C2C12 cells. Differentiated cells were fixed for 15min in 4\% PFA, blocked in 5\%NGS/PBS, and 454 incubated overnight with $\alpha$-alpha-actinin (A7811, Sigma, 1:1000). Primary antibodies were 455 visualized with an Alexa-fluor 594 conjugated secondary antibody (115-585-003, Jackson 456 ImmunoResearch Laboratories); myonuclei were visualized with Hoechst (H3570, Thermofisher, $457 \quad 1: 1000)$

458 Zebrafish. Hand dechorionated larva were fixed in 4\% PFA for $1 \mathrm{hr}$ and directly stained with 459 Alexa-fluor 555 conjugated phalloidin (A34055, Thermofisher, 1:200) for $2 \mathrm{hr}$ room temperature, 460 or blocked in 5\%NGS/PBST for $1 \mathrm{hr}$ and incubated overnight with THSB4 (Abcam, ab211143, 461 1:100) and $\alpha$-Myosin Heavy Chain (F59, Developmental Studies Hybridoma Bank, 1:50). HRPconjugated secondary antibodies in conjunction with the TSA system (Molecular Probes) were 463 used to detect primary antibodies.

464 Imaging. Embryos and larva were imaged with a Zeiss LSM800 confocal microscope; cells were 465 imaged with an inverted Zeiss AxioObserver. Drosophila larva were live-imaged in PBT after $5 \mathrm{~min}$ exposure to diethyl ether. For time-lapse imaging, dechorionated St12 Drosophila embryos were mounted in halocarbon oil and scanned at $2 \mathrm{~min}$ intervals; dechorionated 3-4 somite zebrafish embryos were mounted in $1.5 \%$ low melt agarose supplemented with egg water on a depression slide and scanned at $5 \mathrm{~min}$ intervals. Control and treated samples were prepared and imaged in parallel where possible, and imaging parameters were maintained between

471 treatment groups. Fluorescent intensity and cell morphology measurements were made with

472 ImageJ software.

\section{Locomotion and Startle Response Assays}

474 Drosophila L3 larva locomotion assays were performed as described (Brooks et al., 2016). For 475 zebrafish larva, the Noldus DanioVision and EthoVision software were used to record and 476 quantify larval movement at 6dpf as described (Whittle et al., 2020). Larva were loaded into the 477 DanioVision in 24-well cell culture plates with egg water at random, and acclimated in the 
478 DanioVision box for 5-10 min. The culture plate was automatically tapped after acclimation, and 479 the startle response was recorded for 3s; EthoVision software tracked and recorded fish 480 movement, and reported escape velocity and distance traveled. Statistical analyses were 481 performed only between control and experimental groups assayed on the same day.

482 Hatching Assays

483 0-24hr old embryos were collected on grape agar plates, dechorionated, and genotyped using 484 GFP expression from the TM3, P(twi.Gal4), P(UAS.GFP) balancer. Genotyped embryos were 485 transferred to a grape agar plate, incubated for $24 \mathrm{hr}$ at $25^{\circ} \mathrm{C}$, and then scored for hatching. At 486 least two collections were completed per condition.

487 Clinical sequencing

488 All patients were recruited from St Louis Children's Hospital or Shriners Hospital St Louis. The 489 institutional review board approved this study and all patients and/or parents provided informed consent. Exome sequencing was performed as described in (Sadler et al., 2020) on a cohort of patients with isolated clubfoot and distal arthrogryposis. Variants were validated by Sanger sequencing.

\section{Statistics}

495 Statistical analyses were performed with GraphPad Prism 9 software, and significance was determined with the unpaired, one-tailed student's t-test, one-way ANOVA, or nonparametric tests (for non-Gaussian distributions). Gaussian distribution fit curves were generated with

498 Origin 2019 software. Sample sizes are indicated in the figure legends. Data collection and data 499 analyses were routinely performed by different authors to prevent potential bias. All individuals 500 were included in data analysis.

501 Drosophila. Muscle morphology and size was visualized by Tropomyosin conjugated GFP in 502 hemisegments A2-A8, using 6-10 St16 embryos per genotype. For morphology, muscles were 503 assigned a phenotype (normal, missing, misshapen, elongation defect, attachment site defect), 504 reported as a frequency. Myoblast fusion was quantified by counting the number of lacZ+ 505 myonuclei per hemisegment (A2-A8) in rP298.nlacZ embryos. Fusion Index = \#lacZ nuclei 506 experimental/\#lacZ nuclei control ${ }^{\star} 100$.

507 Zebrafish. Methods for measuring musculoskeletal parameters are shown in Figure 5, and 508 largely reflect those reported in (Chagovetz et al., 2019). To control for day-to-day variability in 509 embryo injections, muscle measurements were first normalized to the daily control and then 510 reported as a percent of control. 
511 C2C12 cells. Absolute myotube length was used for comparisons among treatment groups. 512 Fusion index = \#nuclei in multinucleate myotubes/total nuclei *100. A minimum of 10 fields were 513 quantified per treatment for each parameter. 


\section{References}

Abdul-Hussein, S., Rahl, K., Moslemi, A.R., and Tajsharghi, H. (2013). Phenotypes of myopathy-related beta-tropomyosin mutants in human and mouse tissue cultures. PLoS One 8, e72396.

Avrova, S.V., Karpicheva, O.E., Rysev, N.A., Simonyan, A.O., Sirenko, V.V., Redwood, C.S., and Borovikov, Y.S. (2018). The reason for the low $\mathrm{Ca}(2+)$-sensitivity of thin filaments associated with the Glu41Lys mutation in the TPM2 gene is "freezing" of tropomyosin near the outer domain of actin and inhibition of actin monomer switching off during the ATPase cycle. Biochem Biophys Res Commun 502, 209-214.

Bamshad, M., Van Heest, A.E., and Pleasure, D. (2009). Arthrogryposis: a review and update. J Bone Joint Surg Am 91 Suppl 4, 40-46.

Bamshad, M., Watkins, W.S., Zenger, R.K., Bohnsack, J.F., Carey, J.C., Otterud, B., Krakowiak, P.A., Robertson, M., and Jorde, L.B. (1994). A gene for distal arthrogryposis type I maps to the pericentromeric region of chromosome 9. Am J Hum Genet 55, 1153-1158.

Blau, H.M., Chiu, C.P., and Webster, C. (1983). Cytoplasmic activation of human nuclear genes in stable heterocaryons. Cell 32, 1171-1180.

Borovikov, Y.S., Avrova, S.V., Rysev, N.A., Sirenko, V.V., Simonyan, A.O., Chernev, A.A., Karpicheva, O.E., Piers, A., and Redwood, C.S. (2015). Aberrant movement of $\beta$-tropomyosin associated with congenital myopathy causes defective response of myosin heads and actin during the ATPase cycle. Arch Biochem Biophys 577-578, 11-23.

Borovikov, Y.S., Karpicheva, O.E., Avrova, S.V., Simonyan, A.O., Sirenko, V.V., and Redwood, C.S. (2020). The molecular mechanism of muscle dysfunction associated with the R133W mutation in Tpm2.2. Biochem Biophys Res Commun 523, 258-262.

Borovikov, Y.S., Rysev, N.A., Karpicheva, O.E., Sirenko, V.V., Avrova, S.V., Piers, A., and Redwood, C.S. (2017a). Molecular mechanisms of dysfunction of muscle fibres associated with Glu139 deletion in TPM2 gene. Sci Rep 7, 16797.

Borovikov, Y.S., Simonyan, A.O., Karpicheva, O.E., Avrova, S.V., Rysev, N.A., Sirenko, V.V., Piers, A., and Redwood, C.S. (2017b). The reason for a high $\mathrm{Ca}(2+)$-sensitivity associated with Arg91Gly substitution in TPM2 gene is the abnormal behavior and high flexibility of tropomyosin during the ATPase cycle. Biochem Biophys Res Commun 494, 681-686.

Brooks, D.S., Vishal, K., Kawakami, J., Bouyain, S., and Geisbrecht, E.R. (2016). Optimization of wrMTrck to monitor Drosophila larval locomotor activity. J Insect Physiol 93-94, 11-17.

Bugyi, B., Didry, D., and Carlier, M.F. (2010). How tropomyosin regulates lamellipodial actin-based motility: a combined biochemical and reconstituted motility approach. Embo j 29, 14-26.

Buszczak, M., Paterno, S., Lighthouse, D., Bachman, J., Planck, J., Owen, S., Skora, A.D., Nystul, T.G., Ohlstein, B., Allen, A., et al. (2007). The Carnegie protein trap library: A versatile tool for Drosophila developmental studies. Genetics 175, 1505-1531.

Chagovetz, A.A., Klatt Shaw, D., Ritchie, E., Hoshijima, K., and Grunwald, D.J. (2019). Interactions among ryanodine receptor isotypes contribute to muscle fiber type development and function. Dis Model Mech 13.

Chong, J.X., Talbot, J.C., Teets, E.M., Previs, S., Martin, B.L., Shively, K.M., Marvin, C.T., Aylsworth, A.S., Saadeh-Haddad, R., Schatz, U.A., et al. (2020). Mutations in MYLPF Cause a Novel Segmental Amyoplasia that Manifests as Distal Arthrogryposis. Am J Hum Genet 107, 293-310.

Clarke, N.F., Domazetovska, A., Waddell, L., Kornberg, A., McLean, C., and North, K.N. (2009). Cap disease due to mutation of the beta-tropomyosin gene (TPM2). Neuromuscul Disord 19, 348-351.

Clarke, N.F., and North, K.N. (2003). Congenital fiber type disproportion--30 years on. J Neuropathol Exp Neurol 62, 977-989.

Cortés, F., Daggett, D., Bryson-Richardson, R.J., Neyt, C., Maule, J., Gautier, P., Hollway, G.E., Keenan, D., and Currie, P.D. (2003). Cadherin-Mediated Differential Cell Adhesion Controls Slow Muscle Cell Migration in the Developing Zebrafish Myotome. Developmental Cell 5, 865-876.

Davidson, A.E., Siddiqui, F.M., Lopez, M.A., Lunt, P., Carlson, H.A., Moore, B.E., Love, S., Born, D.E., Roper, H., Majumdar, A., et al. (2013). Novel deletion of lysine 7 expands the clinical, histopathological and genetic spectrum of TPM2-related myopathies. Brain 136, 508-521.

Demontis, F., and Perrimon, N. (2009). Integration of Insulin receptor/Foxo signaling and dMyc activity during muscle growth regulates body size in Drosophila. Development 136, 983-993. 
Dennis, M.J., Ziskind-Conhaim, L., and Harris, A.J. (1981). Development of neuromuscular junctions in rat embryos. Dev Biol 81, 266-279.

Guo, Y., Kronert, W.A., Hsu, K.H., Huang, A., Sarsoza, F., Bell, K.M., Suggs, J.A., Swank, D.M., and Bernstein, S.I. (2020). Drosophila myosin mutants model the disparate severity of type 1 and type 2B distal arthrogryposis and indicate an enhanced actin affinity mechanism. Skelet Muscle 10, 24.

Hardeman, E.C., Bryce, N.S., and Gunning, P.W. (2020). Impact of the actin cytoskeleton on cell development and function mediated via tropomyosin isoforms. Semin Cell Dev Biol 102, 122-131.

Higashijima, S.-i., Okamoto, H., Ueno, N., Hotta, Y., and Eguchi, G. (1997). High-Frequency Generation of Transgenic Zebrafish Which Reliably Express GFP in Whole Muscles or the Whole Body by Using Promoters of Zebrafish Origin. Developmental Biology 192, 289-299.

Janco, M., Bonello, T.T., Byun, A., Coster, A.C., Lebhar, H., Dedova, I., Gunning, P.W., and Böcking, T. (2016). The impact of tropomyosins on actin filament assembly is isoform specific. Bioarchitecture 6, 61-75.

Jarraya, M., Quijano-Roy, S., Monnier, N., Behin, A., Avila-Smirnov, D., Romero, N.B., Allamand, V., Richard, P., Barois, A., May, A., et al. (2012). Whole-Body muscle MRI in a series of patients with congenital myopathy related to TPM2 gene mutations. Neuromuscul Disord 22 Supp/ 2, S137-147.

Jin, J.Y., Wu, P.F., Fan, L.L., Yu, F., Li, J.J., Fan, X.F., Huang, H., Zeng, L., Tang, J.Y., and Xiang, R. (2017). A mutation of beta-tropomyosin gene in a Chinese family with distal arthrogryposis type I. Int J Clin Exp Pathol 10, 11137-11142.

Jing, L., and Zon, L.I. (2011). Zebrafish as a model for normal and malignant hematopoiesis. Dis Model Mech 4, 433-438.

Johnson, A.N., Mokalled, M.H., Valera, J.M., Poss, K.D., and Olson, E.N. (2013). Post-transcriptional regulation of myotube elongation and myogenesis by Hoi Polloi. Development 140, 3645-3656.

Kardon, G. (1998). Muscle and tendon morphogenesis in the avian hind limb. Development 125, 40194032.

Karpicheva, O.E., Simonyan, A.O., Rysev, N.A., Redwood, C.S., and Borovikov, Y.S. (2020). Looking for Targets to Restore the Contractile Function in Congenital Myopathy Caused by GIn(147)Pro Tropomyosin. Int J Mol Sci 21.

Keenan, S.R., and Currie, P.D. (2019). The Developmental Phases of Zebrafish Myogenesis. J Dev Biol 7.

Ko, J.M., Choi, I.H., Baek, G.H., and Kim, K.W. (2013). First Korean family with a mutation in TPM2 associated with Sheldon-Hall syndrome. J Korean Med Sci 28, 780-783.

Lees, J.G., Ching, Y.W., Adams, D.H., Bach, C.T., Samuel, M.S., Kee, A.J., Hardeman, E.C., Gunning, P., Cowin, A.J., and O'Neill, G.M. (2013). Tropomyosin regulates cell migration during skin wound healing. J Invest Dermatol 133, 1330-1339.

Li, S., You, Y., Gao, J., Mao, B., Cao, Y., Zhao, X., and Zhang, X. (2018). Novel mutations in TPM2 and PIEZO2 are responsible for distal arthrogryposis (DA) 2B and mild DA in two Chinese families. 19, 179.

Marston, S., Memo, M., Messer, A., Papadaki, M., Nowak, K., McNamara, E., Ong, R., El-Mezgueldi, M., $\mathrm{Li}, \mathrm{X}$., and Lehman, W. (2013). Mutations in repeating structural motifs of tropomyosin cause gain of function in skeletal muscle myopathy patients. Hum Mol Genet 22, 4978-4987.

Marttila, M., Lehtokari, V.L., Marston, S., Nyman, T.A., Barnerias, C., Beggs, A.H., Bertini, E., CeyhanBirsoy, O., Cintas, P., Gerard, M., et al. (2014). Mutation update and genotype-phenotype correlations of novel and previously described mutations in TPM2 and TPM3 causing congenital myopathies. Hum Mutat 35, 779-790.

Marttila, M., Lemola, E., Wallefeld, W., Memo, M., Donner, K., Laing, N.G., Marston, S., Grönholm, M., and Wallgren-Pettersson, C. (2012). Abnormal actin binding of aberrant $\beta$-tropomyosins is a molecular cause of muscle weakness in TPM2-related nemaline and cap myopathy. Biochem $\mathrm{J}$ 442, 231-239.

Matyushenko, A.M., Shchepkin, D.V., Susorov, D.S., Nefedova, V.V., Kopylova, G.V., Berg, V.Y., Kleymenov, S.Y., and Levitsky, D.I. (2019). Structural and functional properties of aß-heterodimers of tropomyosin with myopathic mutations Q147P and K49del in the $\beta$-chain. Biochem Biophys Res Commun 508, 934-939.

Mokbel, N., Ilkovski, B., Kreissl, M., Memo, M., Jeffries, C.M., Marttila, M., Lehtokari, V.L., Lemola, E., Grönholm, M., Yang, N., et al. (2013). K7del is a common TPM2 gene mutation associated with nemaline myopathy and raised myofibre calcium sensitivity. Brain 136, 494-507. 
Monnier, N., Lunardi, J., Marty, I., Mezin, P., Labarre-Vila, A., Dieterich, K., and Jouk, P.S. (2009). Absence of $\beta$-tropomyosin is a new cause of Escobar syndrome associated with nemaline myopathy. Neuromuscular Disorders 19, 118-123.

Morgan, N.V., Brueton, L.A., Cox, P., Greally, M.T., Tolmie, J., Pasha, S., Aligianis, I.A., van Bokhoven, H., Marton, T., Al-Gazali, L., et al. (2006). Mutations in the embryonal subunit of the acetylcholine receptor (CHRNG) cause lethal and Escobar variants of multiple pterygium syndrome. Am J Hum Genet 79, 390-395.

Mroczek, M., Kabzińska, D., Chrzanowska, K.H., Pronicki, M., and Kochański, A. (2017). A novel TPM2 gene splice-site mutation causes severe congenital myopathy with arthrogryposis and dysmorphic features. J Appl Genet 58, 199-203.

Nose, A., Isshiki, T., and Takeichi, M. (1998). Regional specification of muscle progenitors in Drosophila: the role of the msh homeobox gene. Development 125, 215-223.

Ochala, J., Iwamoto, H., Larsson, L., and Yagi, N. (2010). A myopathy-linked tropomyosin mutation severely alters thin filament conformational changes during activation. Proc Natl Acad Sci U S A 107, 9807-9812.

Ochala, J., Li, M., Ohlsson, M., Oldfors, A., and Larsson, L. (2008). Defective regulation of contractile function in muscle fibres carrying an E41K beta-tropomyosin mutation. J Physiol 586, 2993-3004.

Ohlsson, M., Quijano-Roy, S., Darin, N., Brochier, G., Lacene, E., Avila-Smirnow, D., Fardeau, M., Oldfors, A., and Tajsharghi, H. (2008). New morphologic and genetic findings in cap disease associated with beta-tropomyosin (TPM2) mutations. Neurology 71, 1896-1901.

Robaszkiewicz, K., Ostrowska, Z., Marchlewicz, K., and Moraczewska, J. (2016). Tropomyosin isoforms differentially modulate the regulation of actin filament polymerization and depolymerization by cofilins. Febs j 283, 723-737.

Robinson, P., Lipscomb, S., Preston, L.C., Altin, E., Watkins, H., Ashley, C.C., and Redwood, C.S. (2007). Mutations in fast skeletal troponin I, troponin T, and beta-tropomyosin that cause distal arthrogryposis all increase contractile function. Faseb j 21, 896-905.

Sadler, B., Haller, G., Antunes, L., Nikolov, M., Amarillo, I., Coe, B., Dobbs, M.B., and Gurnett, C.A. (2020). Rare and <em $>$ de novo</em $>$ duplications containing $<$ em $>$ SHOX $</$ em $>$ in clubfoot. Journal of Medical Genetics 57, 851-857.

Shibata, T., Shibata, S., Ishigaki, Y., Kiyokawa, E., Ikawa, M., Singh, D.P., Sasaki, H., and Kubo, E. (2018). Tropomyosin 2 heterozygous knockout in mice using CRISPR-Cas9 system displays the inhibition of injury-induced epithelial-mesenchymal transition, and lens opacity. Mech Ageing Dev 171, 24-30.

Shin, H., Kim, D., and Helfman, D.M. (2017). Tropomyosin isoform Tpm2.1 regulates collective and amoeboid cell migration and cell aggregation in breast epithelial cells. Oncotarget 8, 95192-95205.

Śliwinska, M., Robaszkiewicz, K., Czajkowska, M., Zheng, W., and Moraczewska, J. (2018). Functional effects of substitutions 192T and V95A in actin-binding period 3 of tropomyosin. Biochim Biophys Acta Proteins Proteom 1866, 558-568.

Squire, J.M., Paul, D.M., and Morris, E.P. (2017). Myosin and Actin Filaments in Muscle: Structures and Interactions. Subcell Biochem 82, 319-371.

Sung, S.S., Brassington, A.M., Grannatt, K., Rutherford, A., Whitby, F.G., Krakowiak, P.A., Jorde, L.B., Carey, J.C., and Bamshad, M. (2003). Mutations in genes encoding fast-twitch contractile proteins cause distal arthrogryposis syndromes. Am J Hum Genet 72, 681-690.

Tajsharghi, H., Kimber, E., Holmgren, D., Tulinius, M., and Oldfors, A. (2007a). Distal arthrogryposis and muscle weakness associated with a beta-tropomyosin mutation. Neurology 68, 772-775.

Tajsharghi, H., Ohlsson, M., Lindberg, C., and Oldfors, A. (2007b). Congenital myopathy with nemaline rods and cap structures caused by a mutation in the beta-tropomyosin gene (TPM2). Arch Neurol 64, 1334-1338.

Tajsharghi, H., Ohlsson, M., Palm, L., and Oldfors, A. (2012). Myopathies associated with betatropomyosin mutations. Neuromuscul Disord 22, 923-933.

Vogt, J., Al-Saedi, A., Willis, T., Male, A., McKie, A., Kiely, N., and Maher, E.R. (2020). A recurrent pathogenic variant in TPM2 reveals further phenotypic and genetic heterogeneity in multiple pterygium syndrome-related disorders. Clin Genet 97, 908-914.

Wang, W., Chen, M., Gao, Y., Song, X., Zheng, H., Zhang, K., Zhang, B., and Chen, D. (2018). P2Y6 regulates cytoskeleton reorganization and cell migration of $\mathrm{C} 2 \mathrm{C} 12$ myoblasts via ROCK pathway. Journal of Cellular Biochemistry 119, 1889-1898. 
Whittle, J., Antunes, L., Harris, M., Upshaw, Z., Sepich, D.S., Johnson, A.N., Mokalled, M., SolnicaKrezel, L., Dobbs, M.B., and Gurnett, C.A. (2020). MYH3-associated distal arthrogryposis zebrafish model is normalized with para-aminoblebbistatin. EMBO Mol Med 12, e12356.

681

682

683

Williams, J., Boin, N.G., Valera, J.M., and Johnson, A.N. (2015). Noncanonical roles for Tropomyosin during myogenesis. Development 142, 3440-3452.

684

685

Yang, S., Weske, A., Du, Y., Valera, J.M., Jones, K.L., and Johnson, A.N. (2020). FGF signaling directs myotube guidance by regulating Rac activity. Development 147. 


\section{Figure Legends}

687 Figure 1. TPM2 residues associated with pathogenic variants are conserved. (A) TPM2

688 conservation and sequence structure. TPM2 protein sequence divided into seven quasi repeats 689 and split into $\alpha$ - and $\beta$-bands as described in (Marston et al., 2013). Actin-binding residues are 690 colored blue. Vertical black lines show identical residues between the human (hs) and Drosophila (dm) proteins; gray lines show similar residues. 25 pathogenic TPM2 variants in the coding region have been identified (Marttila et al., 2014). Variants affecting conserved residues are boxed in red; non-conserved pathogenic variants are boxed in black. 3 non-coding pathogenic variants have also been reported (not shown). (B) Conserved pathogenic variants partially cluster to a single topographical position. Diagram showing seven amino acids heptad residues binds actin (shown in blue). 7 conserved pathogenic variants mapped to residues in position ' $g$ '. (C) Transgenic expression assay to characterize myogenic defects in muscles expressing pathogenic TPM2 variants. Stable lines encoding TPM2 variants under UAS control were used to express eight conserved pathogenic in specialized embryonic muscle precursors (founder cells); muscle morphology was analyzed at the end of embryogenesis.

702 Figure 2. TPM2 variants disrupt muscle development in Drosophila. (A,B) Variants 703 expressed under the control of slou.Gal4 caused multiple muscle phenotypes. (A) The diagram 704 depicts the thirty body wall muscles in an embryonic hemisegment; slou.Gal4 expressing 705 muscles are shown in blue [modeled after (Yang et al., 2020)]. Confocal micrographs show 706 Stage 16 embryos that expressed GFP-tagged Drosophila Tropomyosin 2 (Tm2), wild-type 707 human TPM2, or pathogenic TPM2 variants (green), co-labeled with Myosin Heavy Chain 708 (MHC, violet). Two hemisegments are shown for each embryo. Variant-expressing LO1 709 muscles showed multiple phenotypes: rounded muscles (elongation), muscles attached to an 710 incorrect tendon (wrong tendon, white arrows), muscles attached to three tendons (multiple 711 tendons, white arrowheads), muscles absent from a segment (missing), and muscles bent or 712 hook-shaped instead of oblique (misshapen; yellow arrows). (B) Histogram showing phenotypic 713 frequencies ( $n \geq 66$ muscles/genotype from a minimum of 9 embryos/genotype), quantified by a 714 single blinded protocol. (C,D) Variants expressed under the control of nau.Gal4 reduced muscle 715 length. (C) The diagram depicts nau.Gal4 expressing muscles in blue; VO5 muscles show 716 consistent nau.Gal4 expression and VO4 muscles show occasional expression (light blue). 717 Confocal micrographs show Stage 16 embryos that expressed GFP-tagged transgenes, labeled 718 for GFP. Variant-expressing VO5 muscles were short or rounded, but other parameters of 719 muscle morphology were largely normal. E122K expressing muscles showed the strongest 
phenotype (white arrows). (D) Dot plot showing VO5 length quantified as \% of control; each data point represents a single muscle. Error bars, Standard Error of the Mean (SEM). Significance was determined by student's t-test versus Tm2-expressing muscles. Scale bars, $72320 \mu \mathrm{M}$.

724 Figure 3. TPM2 variants disrupt muscle function in Drosophila. (A-C) Larval muscles expressing K49Del and E122K have reduced function. (A) Confocal micrographs of live L3 larva that expressed TPM2.GFP, K49Del.GFP, and E122K.GFP under the control of Mef2.Gal4. Half of the K49Del.GFP expressing larva completely lacked muscles in segment A8. Muscles were also misshapen (arrowheads) or missing (arrow) in variant expressing L3 larva. Scale bars, $500 \mu \mathrm{M}$. (B) Dorsal Oblique muscle length. Each symbol represents average muscle length from 8-14 larva/genotype. Variant expressing muscles in segments A3-A6 were longer than controls. (C) Larval locomotion assays. Variant expressing larva moved less than TPM2 expressing controls. (D,E) K49Del disrupted larval muscle morphology in cross-species rescue assays. (D) Rescue assays were performed by crossing Mef2.Gal4 flies to UAS.TPM2 or UAS.Tm2 flies, and measuring hatching rates of the Mef2>TPM2 and Mef2>Tm2 embryos. All embryos assayed were homozygous for the $T m 2^{D 8-261}$ allele. Hatching rates were determined for control embryos (Gal4-only and UAS-only) and rescued embryos (Mef2>TPM2 and Mef2>Tm2). Rescued embryos showed improved hatching rates compared to controls, but hatching rates were similar between wild-type expressing and K49Del expressing embryos. (E) Tm2 ${ }^{D 8-261}$ Mef2>Tm2.K49Del L1 larva showed muscle defects. Confocal micrographs of live L1 larva. Tm2 $2^{D 8-261}$ Mef2>Tm2.K49Del L1 larva were missing muscles (white arrows), and existing muscles appeared longer than controls. Scale bars, $20 \mu \mathrm{M}$. Significance was determined by 2way ANOVA (B) or unpaired student's t-test $(C)$. (ns) not significant, $\left(^{*}\right) p<0.05$. Error bars, SEM.

744 Figure 4. TPM2 variants disrupt myotube morphogenesis. (A) C2C12 cells transfected with TPM2 variants showed impaired morphology. Confocal micrographs of cells fixed after 7 days in differentiation media, and labeled for $\alpha$-actinin (red) to detect differentiated myotubes, and Hoechst to visualize myonuclei. K49Del and E122K expressing myotubes appeared shorter 748 than controls (vector only and wild-type TPM2), and had fewer myonuclei. Variant expressing 749 myotubes were often rounded (arrows). Scale bars, $20 \mu \mathrm{M}$. (B) Quantification of myoblast fusion. 750 Fusion index represents the number of nuclei in multinucleate myotubes; variant expressing 751 cells fused less than controls. (C) Individual myotubes were measured to quantify cumulative 752 myotube length; variant expressing myotubes were significantly shorter than controls. (D) 753 Myotube length distribution showing the Gaussian distribution fit curve (solid line). Length 
754

755

756

757

758

759

760

761

762

763

764

765

766

767

768

769

770

771

772

773

774

775

776

777

778

779

780

781

782

783

784

785

786

787

distribution of variant expressing myotubes skewed toward shorter lengths. Significance was determined by unpaired student's t-test $(B, C)$ or Mann-Whitney u-test (D). $n \geq 10$ imaging fields per treatment. (ns) not significant, ${ }^{* *}(p<0.01),{ }^{* * *}(p<0.0001)$. Error bars, SEM.

Figure 5. Transient overexpression assays in zebrafish. (A) TPM2 residues associated pathogenic variants are conserved to zebrafish. Vertical black lines show identical residues between the human (hs) and zebrafish ( $d r)$ proteins; gray lines show similar residues. Novel variants (V129A and E139K) are boxed in orange; the recurring variant A155T is boxed in red. (B) One cell stage zebrafish embryos were injected with control or variant encoding mRNAs, and raised under standard conditions to $26 \mathrm{hr}$ post fertilization (hpf) for histological assays or to 6 days post fertilization (dpf) for startle response locomotor assays. (C) Histologic measurements of $26 \mathrm{hpf}$ larva. Individual slow muscle fibers were traced in ImageJ to determine muscle length (magenta line). Somite size was measured 3 times per somite and then averaged to calculate somite length. (D) Pathogenic TPM2 variants caused dose dependent defects in myofiber length and somite length. A gradient of mRNA levels were injected (200pg, 400pg, and $600 \mathrm{pg}$ ), and muscle morphology was assessed at each concentration. Significance was determined by unpaired student's t-test versus wild-type TPM2 injected fish. Each data point represents an individual muscle fiber or somite. (ns) not significant, $\left({ }^{* *}\right) p<0.01,\left({ }^{* * * *}\right) p<0.0001$. Error bars, SEM.

Figure 6. Pathogenic TPM2 variants disrupt myogenesis in zebrafish. (A) Larva expressing K49Del and E122K showed defects in muscle morphogenesis. Confocal micrographs of 26hpf Tg(acta1:GFP) larva injected with TPM2 RNAs at the one cell stage, labeled for F-actin (green) and GFP (violet). Larva that expressed K49Del and E122K had multiple slow myofiber phenotypes including short fibers (red arrows), and myofibers that clustered to single attachment sites at somite boundaries (yellow arrowheads). Larva that expressed the benign variant E273K had morphologically normal myofibers similar to wild-type controls. (B) Larva that expressed K49Del and E122K showed defects in myosepta morphology. Confocal micrographs of 26hpf larva injected with TPM2 RNAs at the one cell stage, labeled for slow myofiber Myosin Heavy Chain (MyHC, green) and the myosepta tendon marker Thrombospondin 4 (THBS4, violet). Larva that expressed K49Del and E122K had multiple phenotypes including tendons that developed in the center of the somite (red arrowhead), bifurcated myosepta (orange arrowheads), and myosepta with broken thrombospondin expression (yellow arrowheads). (C) Gaussian distribution fit curves show K49Del and E122K larva had significantly shorter slow myofibers than E273K larva, and E122K had significantly smaller somites. Myofiber length is the average fiber length per somite. $n \geq 48$ somites per treatment. (D) Violin plot shows K49Del larva 
had significantly fewer slow myofibers per somite than E273K larva. $n \geq 48$ somites per treatment. (E) Violin plot shows E122K larva had significantly more tendon defects per animal than E273K larva. $\mathrm{n} \geq 11$ fish per treatment. Scale bars, $20 \mu$ M. Significance was determined by Mann-Whitney u-test $(C)$, and unpaired student's t-test (D, E). (ns) not significant, $\left({ }^{*}\right) p<0.05$, $\left.\left.{ }^{* * *}\right) p<0.001,{ }^{* * * *}\right) p<0.0001$. Error bars, SEM.

793 Figure 7. TPM2 variants were identified in patients with clubfoot and distal

794 arthrogrypsosis. (A) Clinical features of Patient I, diagnosed with bilateral clubfoot (shown here 795 before treatment). (B,C) Clinical features of Patient II and Patient III, diagnosed with DA1. 796 Photos for patient II show clubfoot before treatment, and photos of Patient III show bilateral 797 clubfoot after treatment. Individual III also developed lower extremity weakness as a child. 798 Patient I and Patient II are heterozygous for the novel variants V129A and E139K, respectively; 799 Patient III is heterozygous for the recurring variant A155T.

800 Figure 8. Novel and recurring TPM2 variants disrupt myogenesis in zebrafish. (A) Larva 801 expressing V129A, E139K, and A155T showed defects in muscle morphogenesis. Confocal 802 micrographs of 26hpf $\operatorname{Tg}$ (acta1:GFP) larva injected with TPM2 RNAs at the one cell stage, 803 labeled for F-actin (green) and GFP (violet). Larva that expressed V129A, E139K, and A155T 804 had multiple slow myofiber phenotypes including short fibers (red arrows), and fewer myofibers 805 than larva that expressed the benign variant E273K. (B) Larva that expressed V129A, E139K, and A155T showed defects in myosepta morphology. Confocal micrographs of 26hpf larva injected with TPM2 RNAs at the one cell stage, labeled for slow myofiber Myosin Heavy Chain (MyHC, green) and the myosepta tendon marker Thrombospondin 4 (THBS4, violet). Larva that expressed V129A, E139K, and A155T had multiple phenotypes including tendons that developed in the center of the somite (red arrowhead), and myosepta with broken thrombospondin expression (yellow arrowheads). (C) Gaussian distribution fit curves show V129 and A155T larva had significantly shorter slow myofibers than E273K larva, and that somite size was significantly different in E139K and A155T larva. Myofiber length is the average fiber length per somite. $n \geq 44$ somites per treatment. (D) Violin plot shows A155T larva had significantly fewer slow myofibers per somite than E273K larva. $n \geq 44$ somites per treatment. (E) Violin plot shows V129A, E139K, and A155T larva had significantly more tendon defects per animal than E273K larva. $\mathrm{n} \geq 12$ fish per treatment. Scale bars, $20 \mu \mathrm{M}$. Significance was determined by Mann-Whitney u-test $(C)$, and unpaired student's t-test (D, E). (ns) not significant, $\left({ }^{*}\right) p<0.05,\left({ }^{* * *}\right) p<0.001,\left({ }^{* * *}\right) p<0.0001$. Error bars, SEM. 
820 Figure 9. TPM2 variants affect muscle function in zebrafish. (A) Automated tracking of the 821 startle response in 6dpf larva. Ethovision imaging software tracked larva for 3s after a 822 mechanical stimulus. Red lines show the locomotor path of representative larva. End points are 823 marked by a square. (B) Larva that expressed V129A, E139K, and A155T had reduced startle 824 responses compared to E273K larva. Swim distance and escape velocity of TPM2 variant 825 injected larva were reported by imaging software, and normalized to wild-type TPM2 injected 826 larva. Significance was determined by student's t-test versus E273K larva. Each data point 827 represents an individual fish. (ns) not significant, $\left({ }^{*}\right) p<0.05,\left({ }^{* *}\right) p<0.01$. Error bars, SEM. 


\section{Movie Captions}

829 Movie 1. K49Del expressing myotubes fail to elongate. LO1 myotubes are pseudocolored 830 blue (WT) or red (K49Del). The K49Del expressing LO1 myotube initiated elongation, but then 831 retracted and failed to reach the muscle attachment site.

832 Movie 2. K49Del expressing myotubes develop a third leading edge. LO1 myotubes are 833 pseudocolored blue (WT) or red (K49Del). The K49Del expressing LO1 myotube initiated 834 elongation with two leading edges, but then developed a third leading edge that appeared to 835 attach to a tendon cell by the end of the movie. Notice a motile cell pinched off of the mutant 836 LO1 and left the filed of view (psuedocolored yellow). 
Table 1. Properties of pathogenic TPM2 variants characterized in vivo.

\begin{tabular}{|c|c|c|c|}
\hline Variant & Diagnosis & Molecular Phenotype & Reference \\
\hline K7Del & $\mathrm{DA}, \mathrm{NM}$ & increased $\mathrm{Ca}^{2+}$ sensitivity & $\begin{array}{l}\text { (Marttila et al., 2014) } \\
\text { (Davidson et al., 2013) } \\
\text { (Marston et al., 2013) } \\
\text { (Mokbel et al., 2013) }\end{array}$ \\
\hline $\mathrm{E} 41 \mathrm{~K}$ & DA, NM & reduced $\mathrm{Ca}^{2+}$ sensitivity & $\begin{array}{l}\text { (Avrova et al., 2018) } \\
\text { (Marttila et al., 2014) } \\
\text { (Marttila et al., 2012) } \\
\text { (Ochala et al., 2008) } \\
\text { (Tajsharghi et al., 2007b) }\end{array}$ \\
\hline K49Del & $\mathrm{CM}$ & $\begin{array}{l}\text { increased } \mathrm{Ca}^{2+} \text { sensitivity } \\
\text { reduced actin affinity }\end{array}$ & $\begin{array}{l}\text { (Matyushenko et al., 2019) } \\
\text { (Marttila et al., 2012) } \\
\text { (Ohlsson et al., 2008) }\end{array}$ \\
\hline R91G & DA & $\begin{array}{l}\text { increased } \mathrm{Ca}^{2+} \text { sensitivity } \\
\text { reduced actin affinity }\end{array}$ & $\begin{array}{l}\text { (Robinson et al., 2007) } \\
\text { (Sung et al., 2003) }\end{array}$ \\
\hline E122K & CFTD & unknown & $\begin{array}{l}\text { (Marttila et al., 2014) } \\
\text { (Tajsharghi et al., 2012) }\end{array}$ \\
\hline R133P & CFTD & unknown & (Marttila et al., 2014) \\
\hline R133W & $\begin{array}{l}\text { DA, EVMPS, } \\
\text { NM }\end{array}$ & reduced $\mathrm{Ca}^{2+}$ sensitivity & $\begin{array}{l}\text { (Vogt et al., 2020) } \\
\text { (Borovikov et al., 2020) } \\
\text { (Ko et al., 2013) } \\
\text { (Ochala et al., 2010) } \\
\text { (Tajsharghi et al., 2007a) }\end{array}$ \\
\hline N202K & $\mathrm{CM}$ & unknown & $\begin{array}{l}\text { (Jarraya et al., 2012) } \\
\text { (Ohlsson et al., 2008) }\end{array}$ \\
\hline V129A & clubfoot & unknown & this study \\
\hline E139K & DA & unknown & this study \\
\hline A155T & DA & unknown & $\begin{array}{l}\text { this study } \\
\text { (Jin et al., 2017) }\end{array}$ \\
\hline
\end{tabular}

(CM) Cap Myopathy, (CFTD) Congenital Fiber Type Disproportion, (DA) Distal Arthrogrypsosis, (EVMPS) Escobar Variant of Multiple Pterygium Syndrome, (NM) Nemaline Myopathy 
Table 2. TPM2 variants of uncertain significance.

\begin{tabular}{lll} 
Variant & Clinical significance* & Condition \\
\hline D2V & likely pathogenic & not provided \\
Q9P & likely pathogenic & inborn genetic disease \\
S61P & likely pathogenic & NM4 \\
K77R & conflicting interpretations & DA1A \\
R90H ${ }^{* *}$ & likely pathogenic & NM \\
Q93H & likely pathogenic & not provided \\
Q103K & likely pathogenic & DA2B \\
E115Del & uncertain & DA1A \\
K128E & uncertain & DA1A \\
H153Q & uncertain & DA1A \\
R178L & uncertain & DA1A \\
D212G & uncertain & DA1A \\
L227V & uncertain & DA1A \\
Q276E & uncertain & \\
\hline
\end{tabular}

*as reported in ClinVar

**all variants showed germline inheritance except $\mathrm{R} 90 \mathrm{H}$ (maternal inheritance) (DA1A) Distal Arthrogrypsosis, type 1a (DA2B) Distal Arthrogrypsosis, type 2b (NM) Nemaline Myopathy 
bioRxiv preprint doi: https://doi.org/10.1101/2021.05.27.445925; this version posted May 27, 2021. The copyright holder for this preprint (which was not certified by peer review) is the author/funder. All rights reserved. No reuse allowed without permission.

Figure $1 \mathrm{~A}$

1

abcdefgabcdefgabcdefgabcdef

hs MDAIKKKMQMLKLDKENAIDRAEQAEA

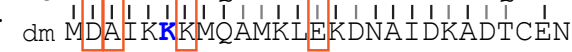
D2V K7Del D14V

A3G

47

efgabcdefgabcdefgabc hs QKKLKGTEDEVEKYSESVKE dm EKKFVEVEIDLVTA KELLEK K49Del G52Dup S61P

cdefgabcdefgabcdefg

87 hs SLNRRIQLVEEELDRAQER dm TQNRKVÍQ I I I I I I I I KSEER

R91G Q93R/H Q103R

gabcdefgabcdefgabcde

126 hs GMKVIENRAMKDEEKMETRE

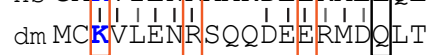
K128E R133P/W E139Del

L143P

defgabcdefgabcdefgab

165 hs VARKLVILEGELERSEERAE dm VSRKLAFVEDELEVAEDRVR

E181K

bcdefgabcdefgabcdef

205

hs KSLEAQADKYSTKEDKYEE

am KSLEVSEEKANQR I I I I I I I KR

Q210* Q218Del

fgabcdefgabcdefgabcd

244 hs RSVAKLEKTIDDLEDEVYAQ dm KQVKRLLKEVDRLEDEL I IN

Y261C

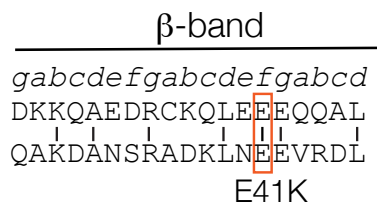

defgabcdefgabcdefgab AQEKLEQAEKKATDAEADVA 1
ANTELEEKEKLLTATESEVA

abcdefgabcdefgabcdef LATALQKLEEAEKAADESER STTAQQKLLEATCSADENNR

\section{E117K E122K}

fgabcdefgabcdefgabc MQLKEAKHIAEDSDRKYEE

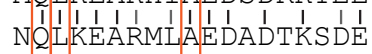

Q147P A155TN

L148P

cdefgabcdefgabcdefga VAESKCGDLEEELKIVTNNL

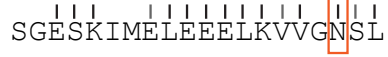
$\mathrm{N} 2 \mathrm{O} 2 \mathrm{~K}$

gabcdefgabcdefgabcde EIKLLTEKLKEAETRAEFAE

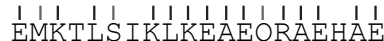

efgabcdefgabcdefgabcd KMKYKAISEELDNALNDITSL 1 I I I I I I I I I I I
KDRYESLADEMDSTELAG repeat

1

2

3

4

5

6

B

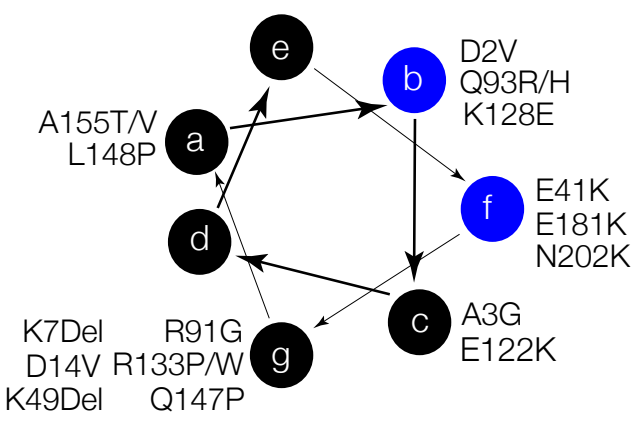

C Transgenic phenotyping assay

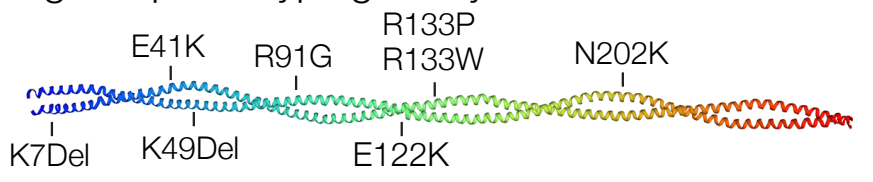

TPM2 hORF variants

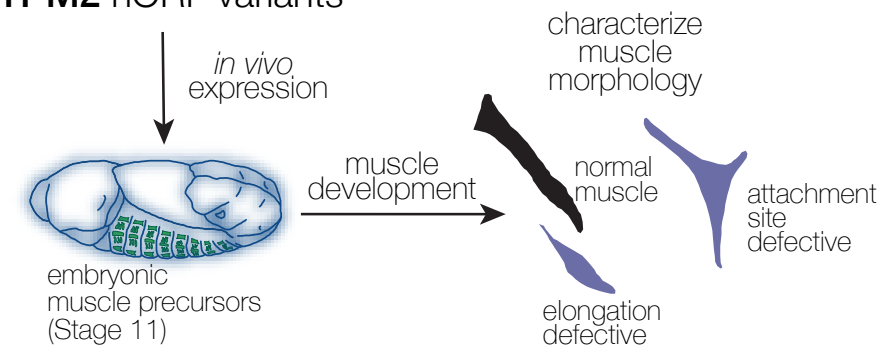


Figure 2

A
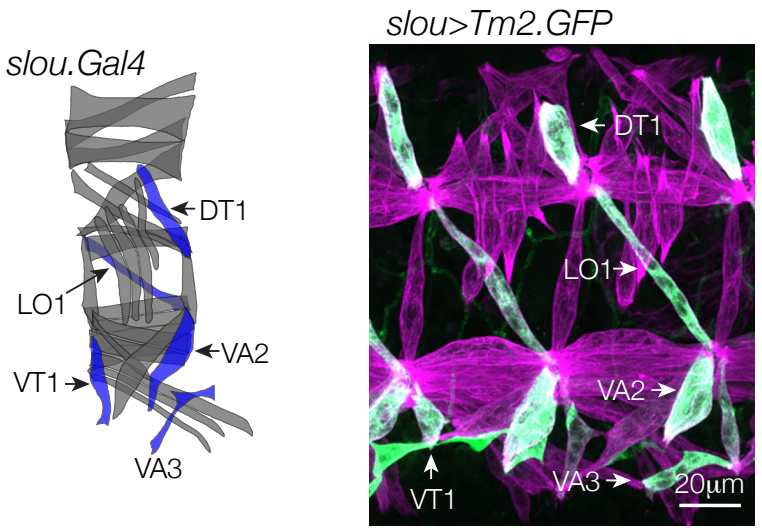

slou>TPM2.GFP

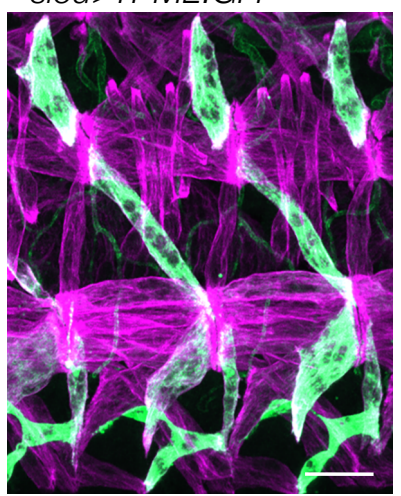

slou>TPM2.E41K.GFP

slou>TPM2.K49Del.GFP

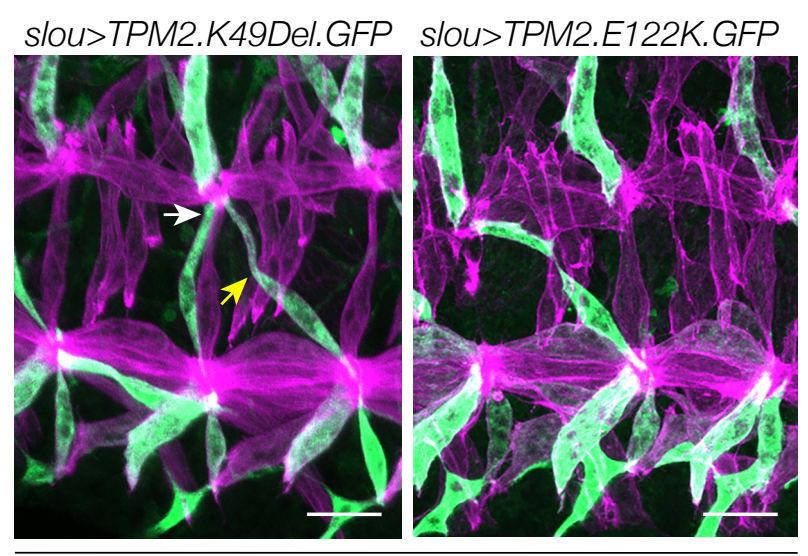

B

GFP/MHC

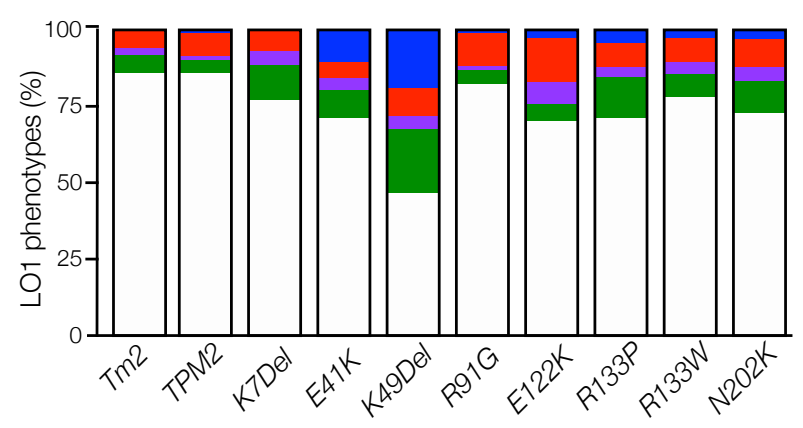

GFP/MHC

C

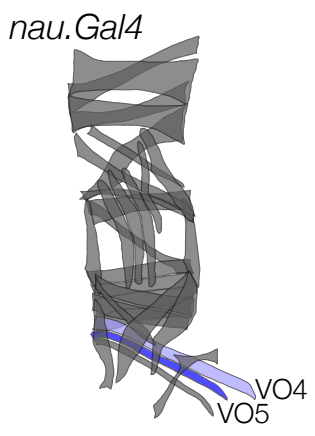

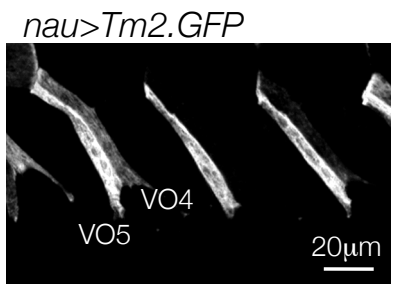

nau>TPM2.K49Del.GFP

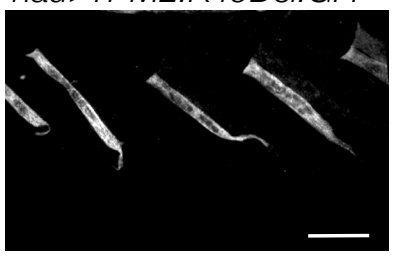

nau>TPM2.GFP

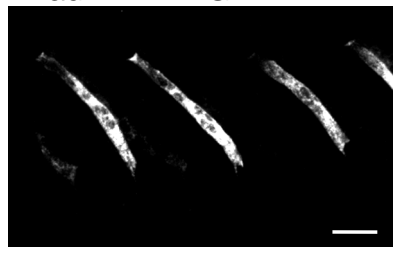

nau>TPM2.E122K.GFP

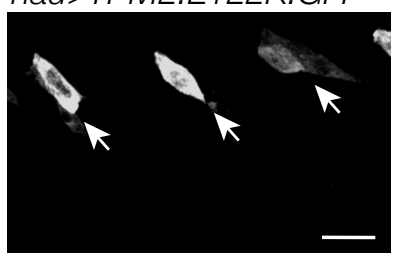

nau>TPM2.E41K.GFP

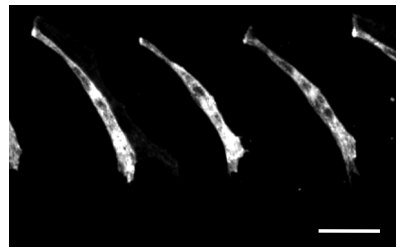

nau>TPM2.N202K.GFP

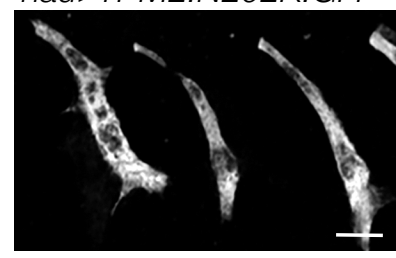

GFP

$\mathrm{D}$

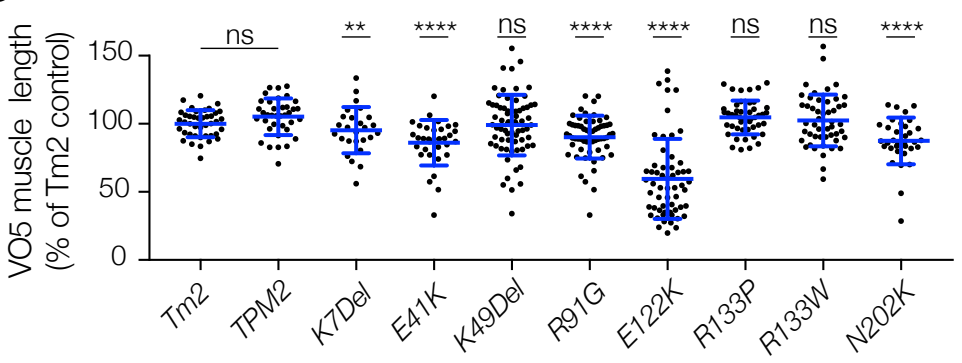


Figure 3

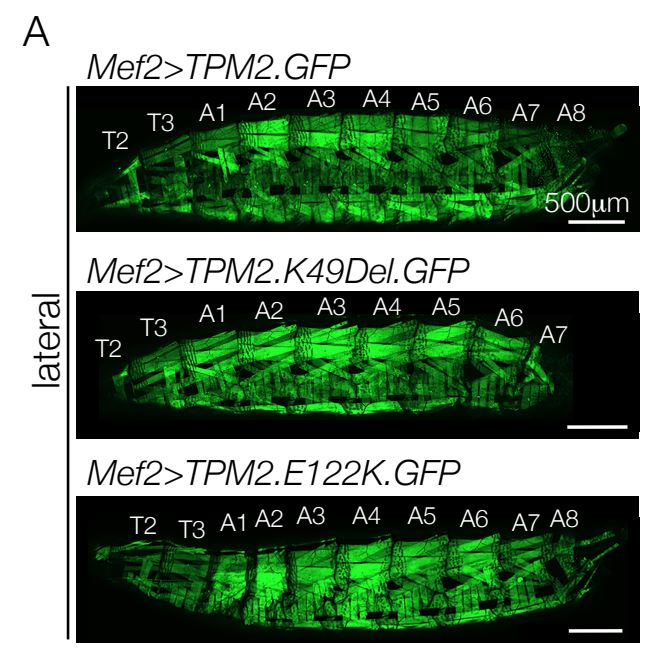

$\mathrm{B}$

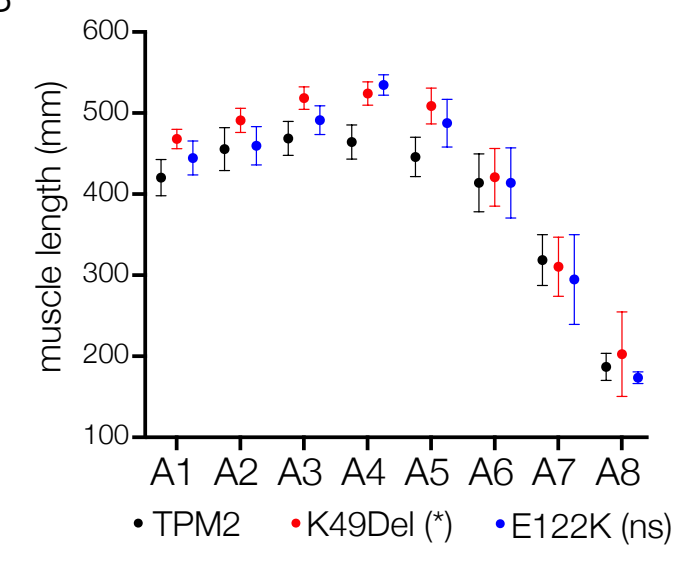

D

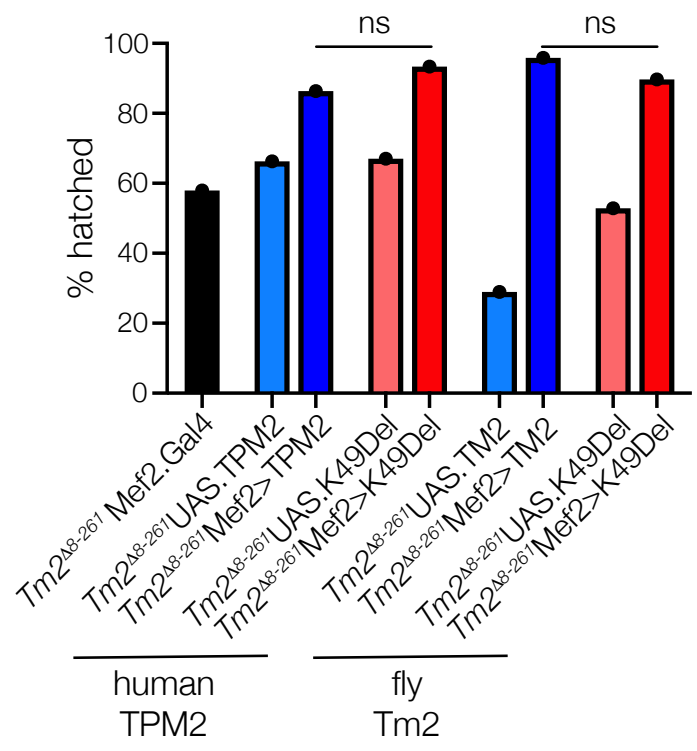

Mef2>TPM2.GFP

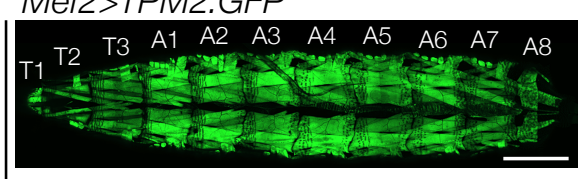

Mef2>TPM2.K49Del.GFP

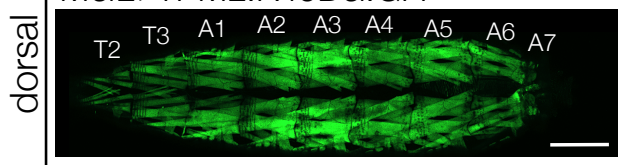

Mef2>TPM2.E122K.GFP

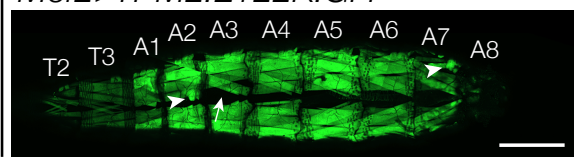

C

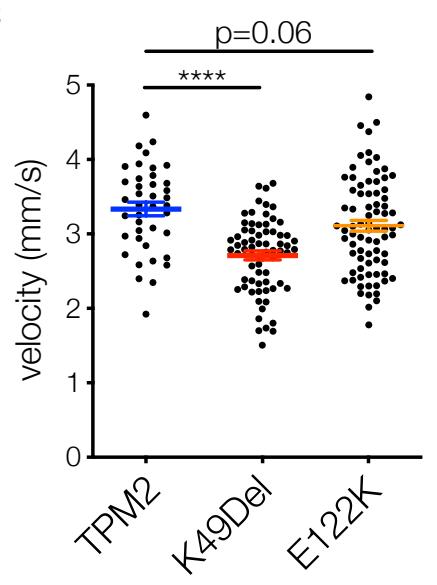

E Tm2 $2^{\Delta 8-261}$

Mef2>TM2.GFP

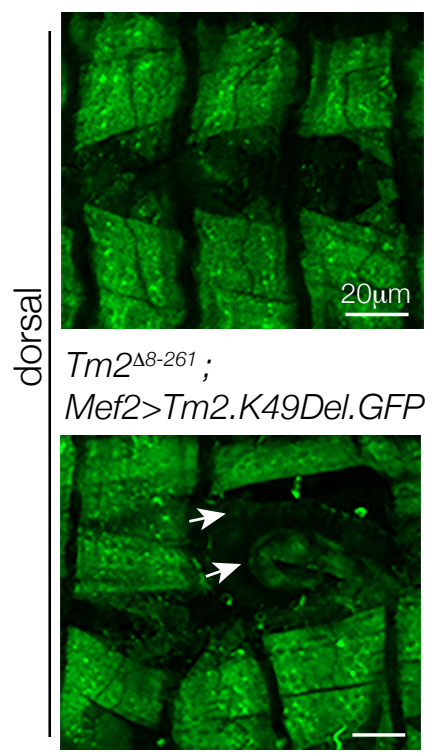


Figure 4

A
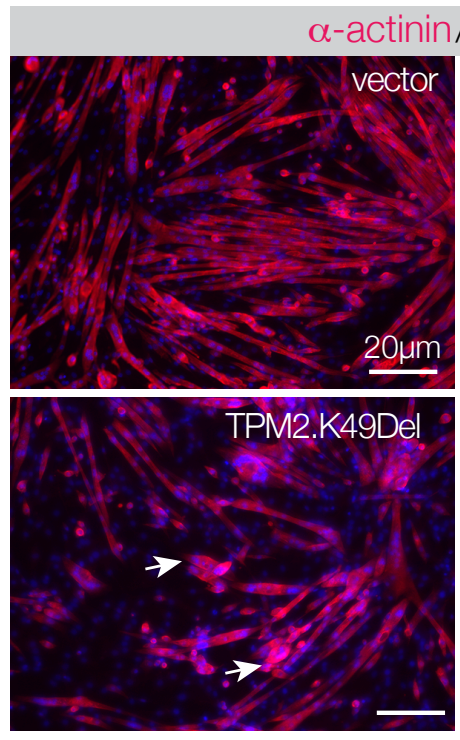

B

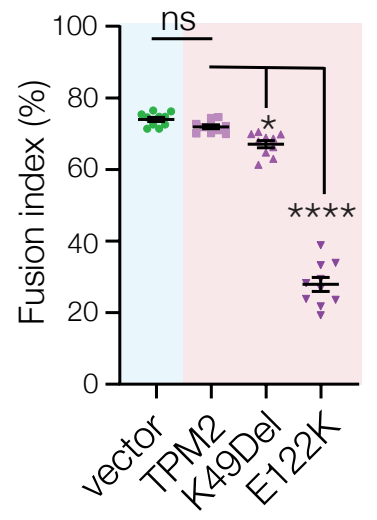

$\mathrm{C}$

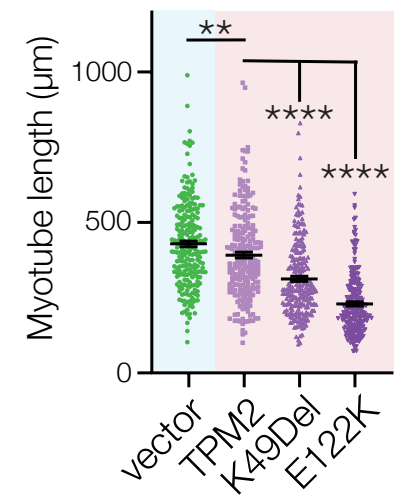

D
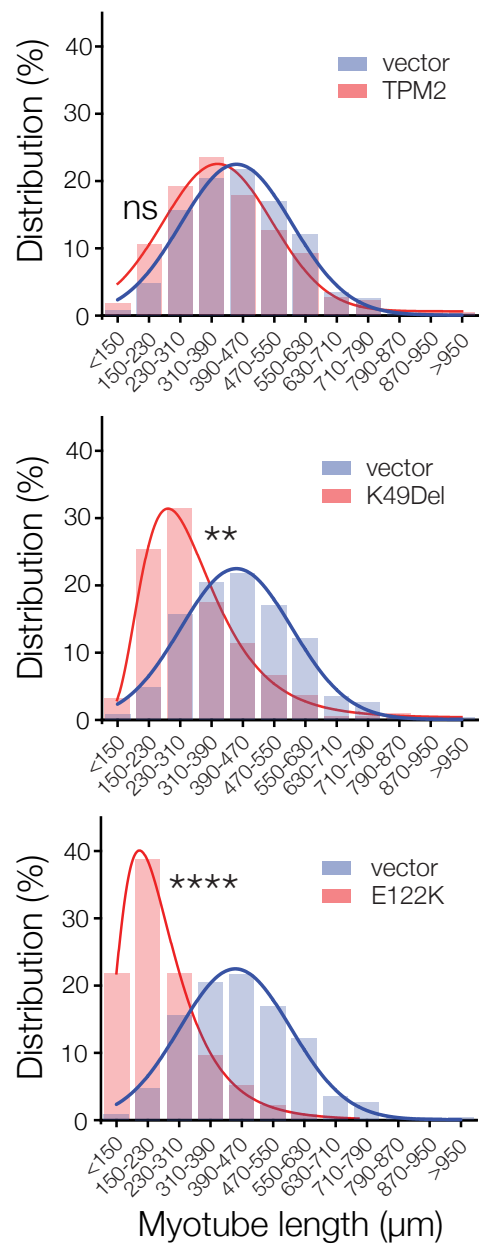
bioRxiv preprint doi: https://doi.org/10.1101/2021.05.27.445925; this version posted May 27, 2021. The copyright holder for this preprint (which was not certified by peer review) is the author/funder. All rights reserved. No reuse allowed without permission.

Figure 5

A

87

126

165

205

244

$\overline{a b c d e f g a b c d e f g a b c d e f g a b c d e f}$ hs MDAIKKKMQMLKLDKENA IDRAEQAEA

1
1
dr MEAIKKKMMQMLKLDKENAIDRAEQAEI

efgabcdefgabcdefgabc

47 hs QKKLKGTEDEVEKYSESVKE

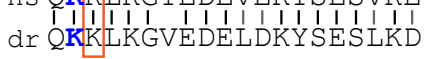
K49Del

cdefgabcdefgabcdefg hs SLNRRIQLVEEELDRAQER

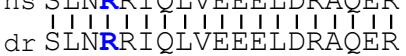

gabcdefgabcdefgabcde hs GMKVIENRAMKDEEKMELQE

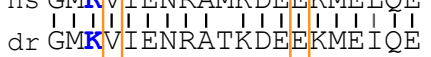

V129A

E139K

Patient I

Patient II

defgabcdefgabcdefgab hs VARKLVI LEGELERSEERAE

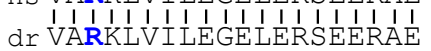

bcdefgabcdefgabcdef hs KSLEAQADKYSTKEDKYEE dr KSLEAQAEKYSTKEDKYEE

fgabcdefgabcdefgabcd hs RSVAKLEKTIDDLEDEVYAQ dr RSVAKLEKTIDDLEDEVYAL

\begin{tabular}{|c|c|}
\hline$\beta$-band & repeat \\
\hline 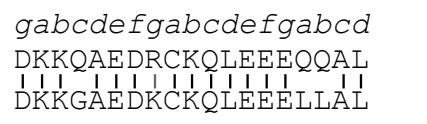 & 1 \\
\hline 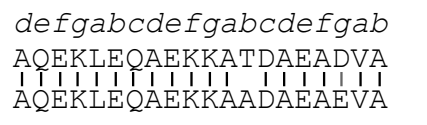 & 2 \\
\hline 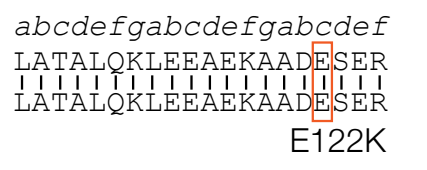 & 3 \\
\hline $\begin{array}{c}\text { fgabcde fgabcdefgabc } \\
\text { MQLKEAKHIAEDSDRKYEE } \\
\text { II I I I I I I I I I I I I I I I } \\
\text { MQLKEAKHAEEADRKYEE } \\
\text { A155T } \\
\text { Patient III }\end{array}$ & 4 \\
\hline $\begin{array}{l}\text { cdefgabcdefgabcdefga } \\
\text { VAESKCGDLEEELKIVTNNL } \\
111 \text { I } 1111111111111 \\
\text { VAEAKSGDLEEELKNVTNNL }\end{array}$ & 5 \\
\hline 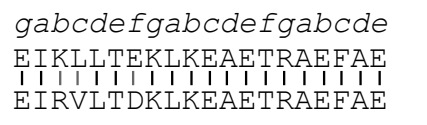 & 6 \\
\hline 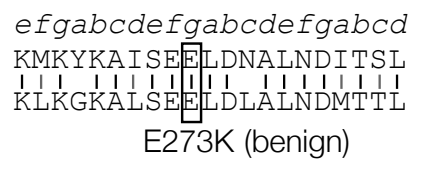 & 7 \\
\hline
\end{tabular}
DKKOAFDRCKOLEEEOQAT III I I I I I I I I I I I I
DKKGAEDKCKQLEEELAL 1 4 5 VAESKCGDLEEELKIVTNNL VAEAKSGDLEEELKNVTNNL

(benign)

B

Inject TPM2 mRNA

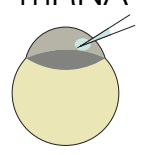

histologic assays

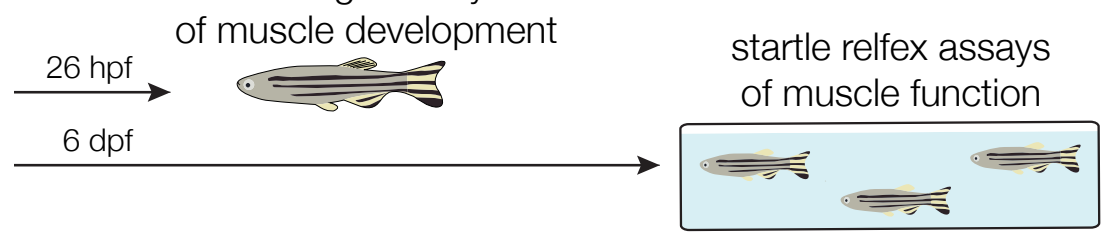

C muscle parameters

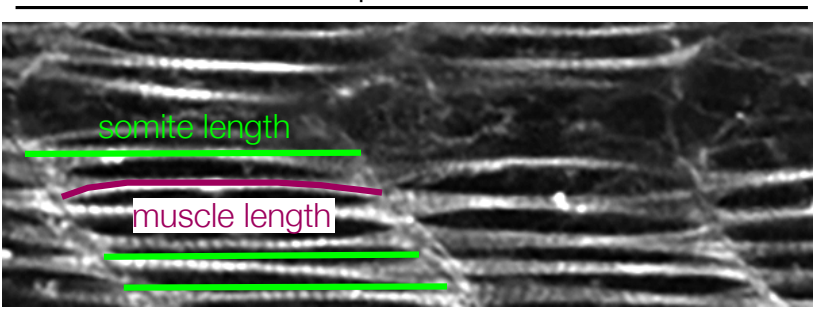

D
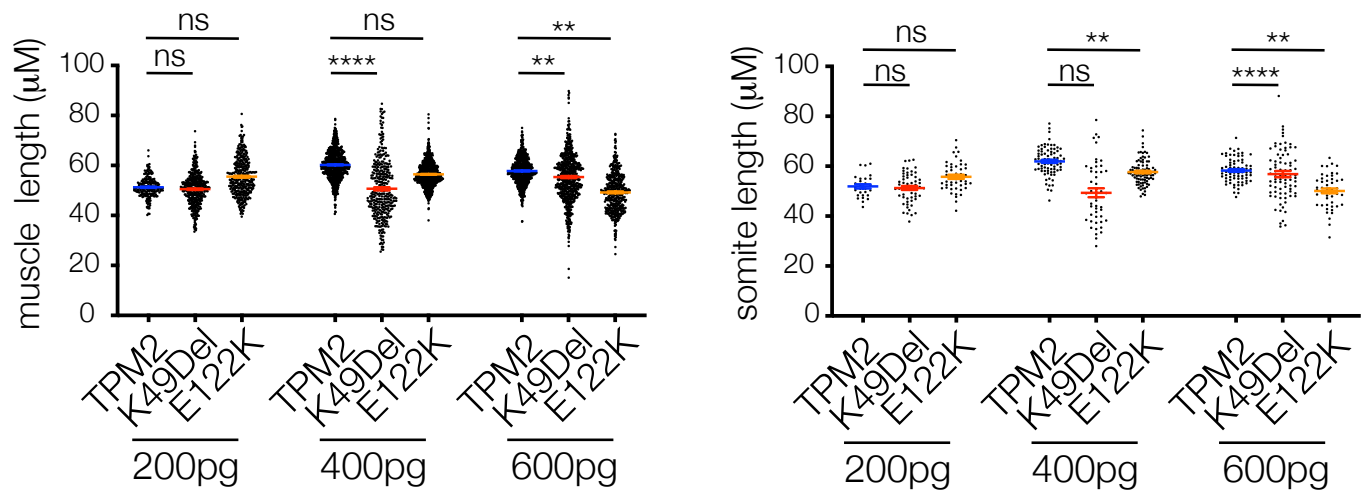
Figure 6

A

F-actin/Tg(acta1:GFP)
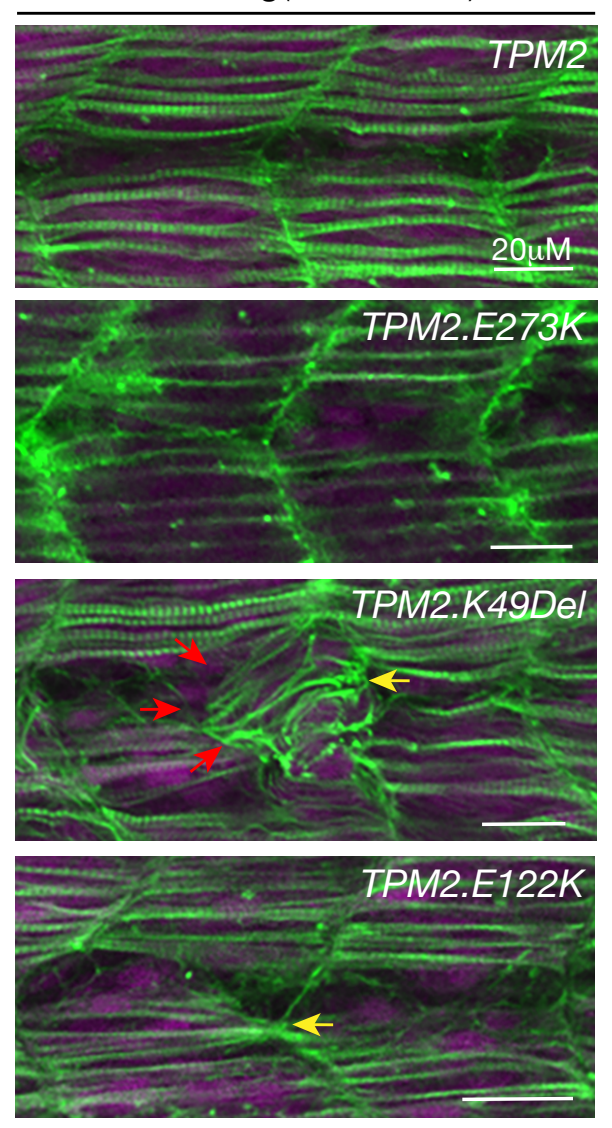

C
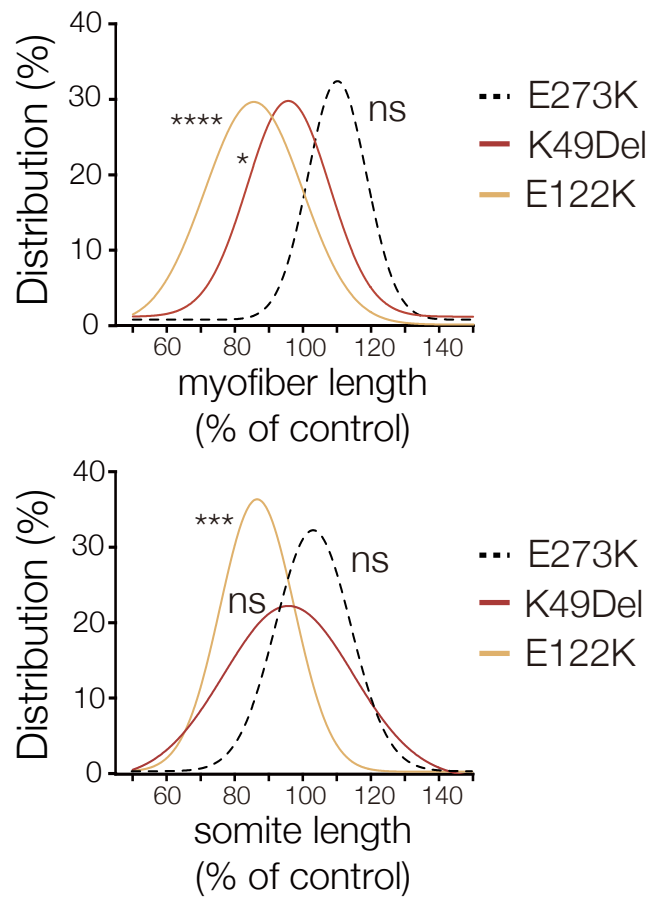

B
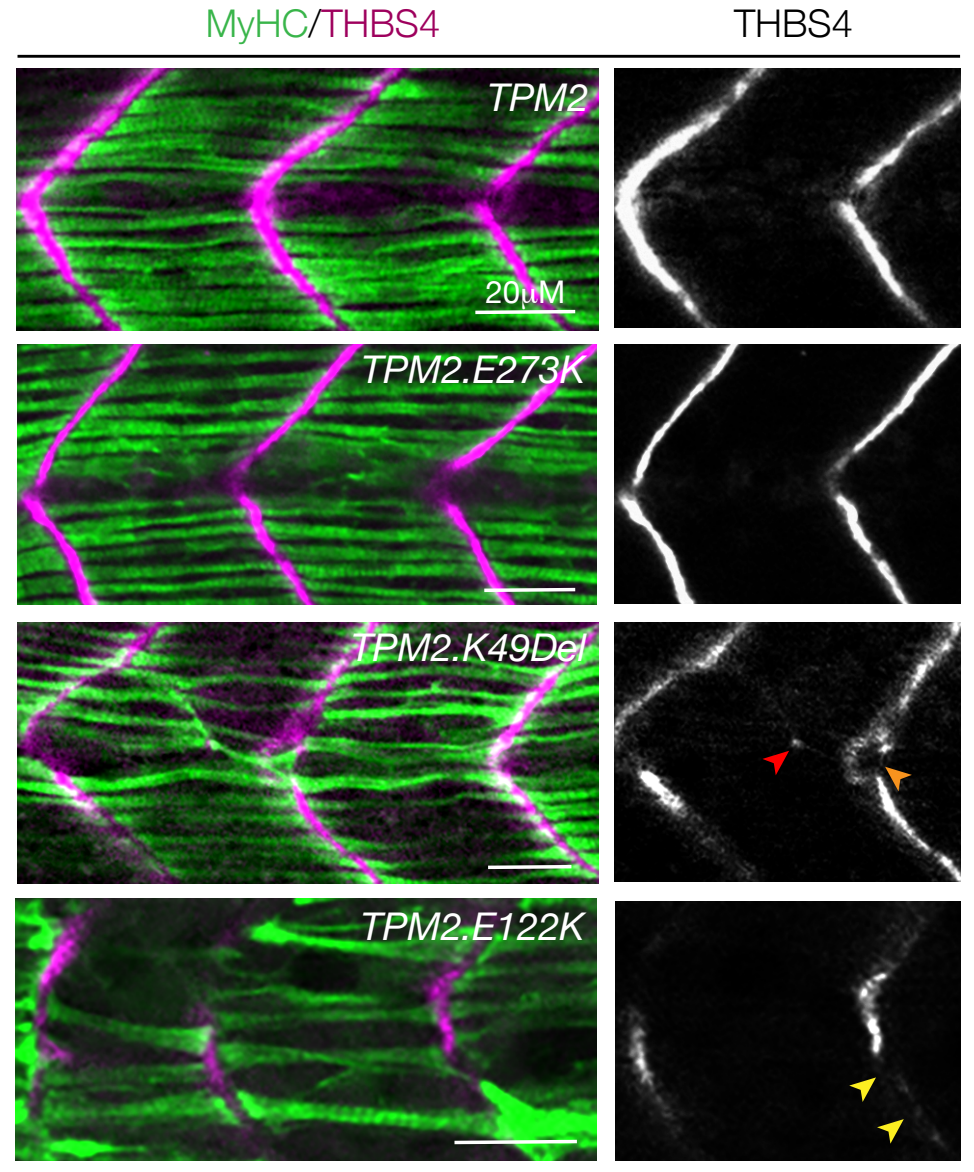

D

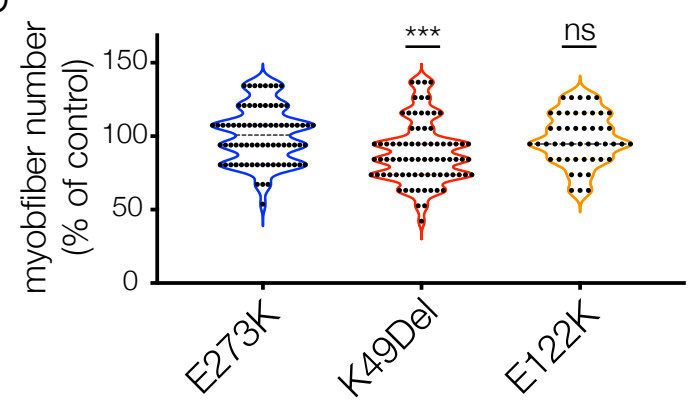

E

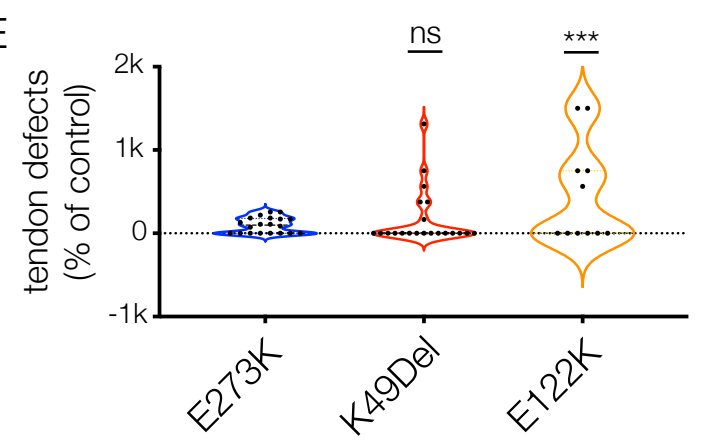


bioRxiv preprint doi: https://doi.org/10.1101/2021.05.27.445925; this version posted May 27, 2021. The copyright holder for this preprint (which was not certified by peer review) is the author/funder. All rights reserved. No reuse allowed without permission.

Figure 7

A

Patient I (V129A)

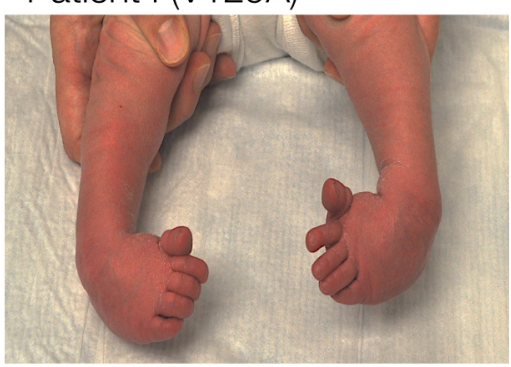

B Patient II (E139K)
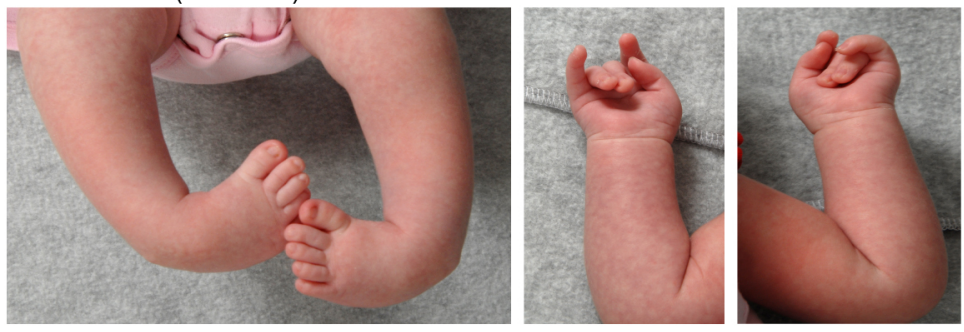

C

\section{Patient III (A155T)}
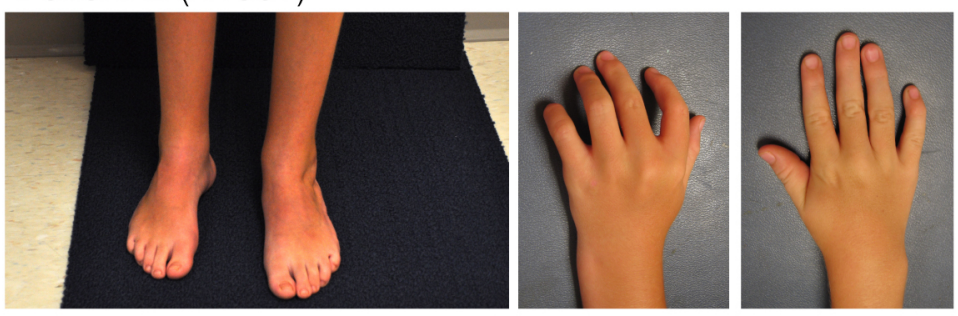
Figure 8

A

F-actin/Tg(acta1:GFP)
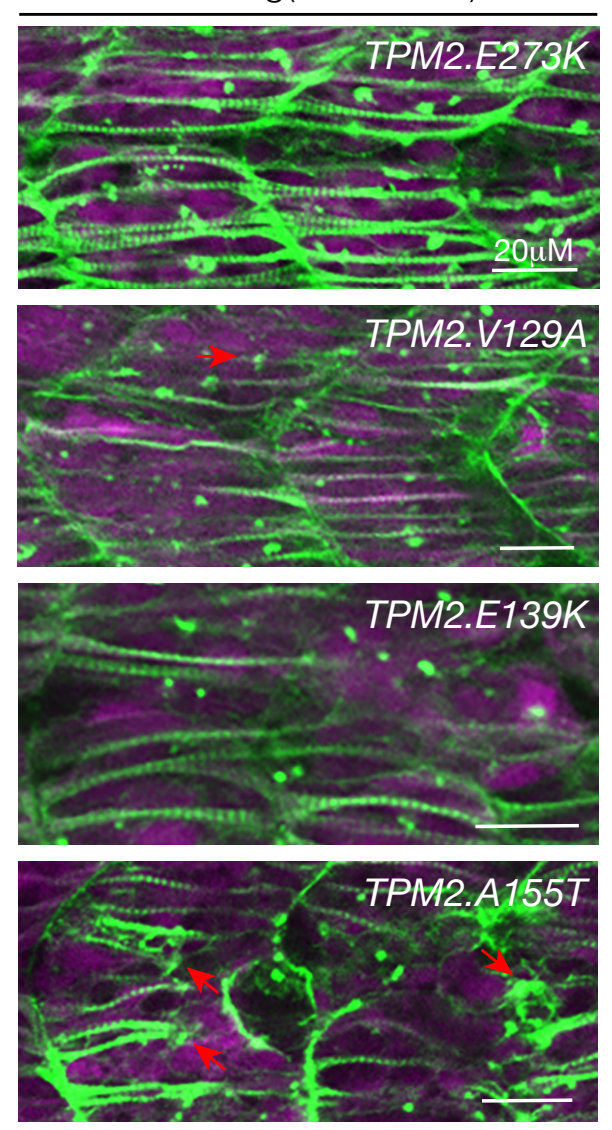

C
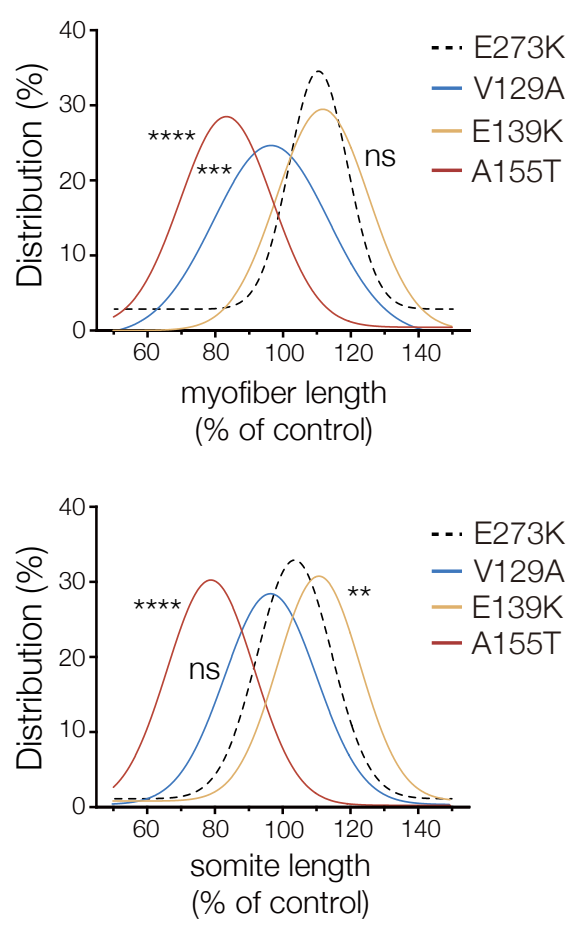

B
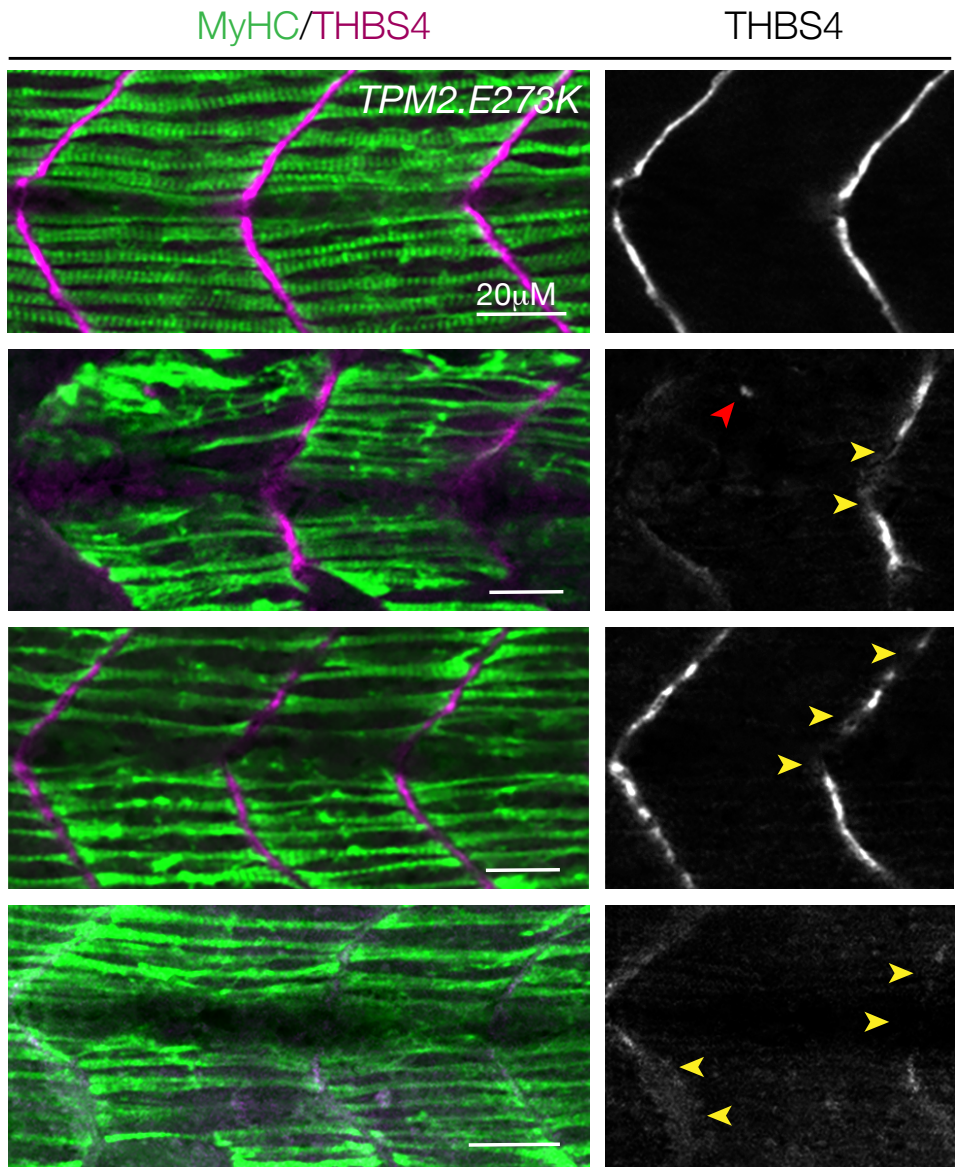

D

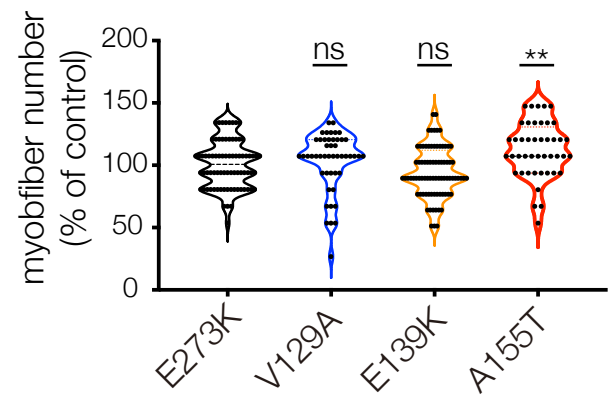

E

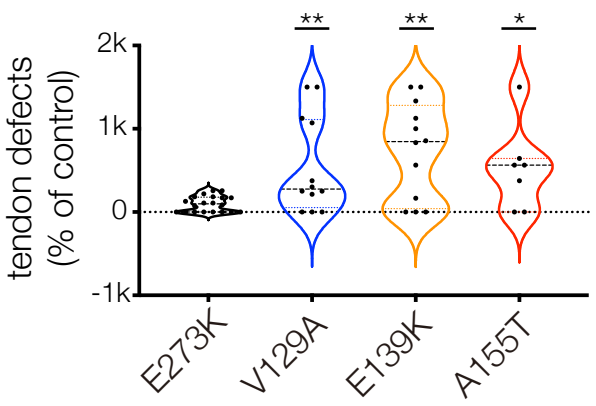


Figure 9

A
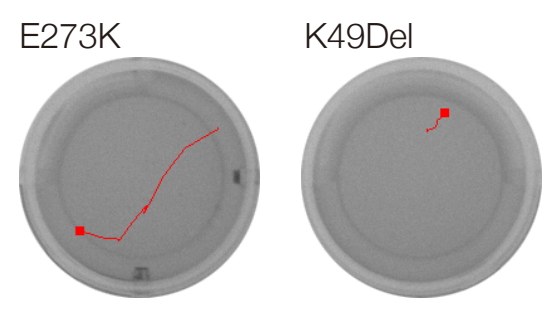

E122K

V129A

E139K
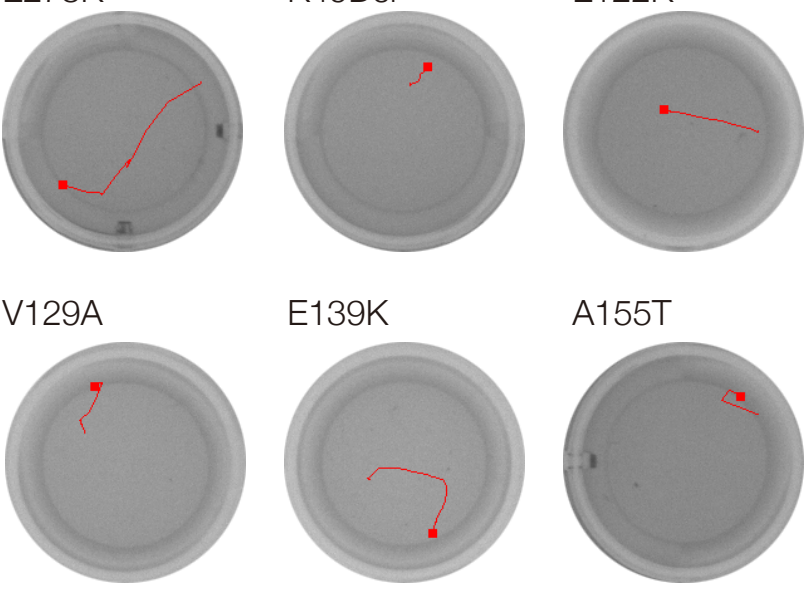

A155T

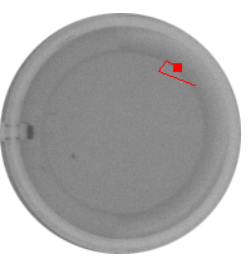

B
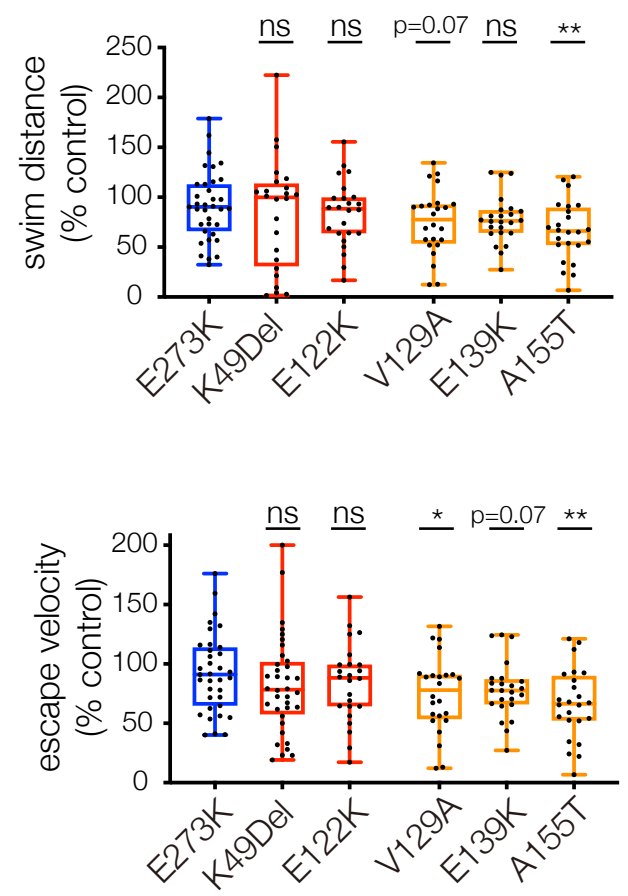